Collection SFN 8 (2007) 115-139

(C) EDP Sciences, Les Ulis

DOI: $10.1051 / \mathrm{sfn}: 2007010$

\title{
Fluctuations de membranes : étude par diffusion de rayonnement
}

\author{
T. Charitat ${ }^{1}, \mathrm{~S}$. Lecuyer ${ }^{1}$ et J. Daillant ${ }^{2}$ \\ ${ }^{1}$ Institut Charles Sadron (UPR 22), CNRS-ULP*, 6 rue Boussingault, BP. 40016, \\ 67083 Strasbourg Cedex, France \\ ${ }^{2}$ Service de Chimie Moléculaire/LIONS, CEA-Saclay, Bât. 125, 91191 Gif-sur-Yvette Cedex, \\ France
}

\begin{abstract}
Résumé. Les molécules amphiphiles s'auto-associent en solutions pour former des structures complexes. Les membranes sont un exemple de ce type de structures. Ce sont des objets bidimensionnels fluctuants qui présentent des propriétés physiques remarquables, mettant en jeu une gamme d'échelles spatiales très large : échelle moléculaire nanométrique pour l'épaisseur de la membrane, de la centaine de nanomètre à la dizaine de microns pour la taille des vésicules, et jusqu'au centimètre pour lasurface des membranes supportées. Ceci explique la grande variété de techniques expérimentales utilisées pour leur étude : microscopie optique classique (contraste de phase...) et interférentielle (RICM), microscopie de fluorescence, microscopie à force atomique (AFM), diffusion de rayonnement. Cette dernière technique permet en particulier d'obtenir des informations uniques sur la structure moléculaire des membranes, mais aussi sur leurs fluctuations à des échelles submicroniques. Dans ce cours nous nous focaliserons sur ce dernier point, essentiellement au travers d'exemples d'études des fluctuations par diffusion de neutrons et de rayons X.
\end{abstract}

\section{INTRODUCTION}

Les membranes de tensioactifs sont des systèmes qui entrent en jeu dans des domaines aussi divers que l'industrie cosmétique (émulsions), pharmaceutique (transport et relargage de médicaments), ou la fonctionnalisation de surfaces (biocapteurs, catalyse...)... D'un point de vue plus fondamental, les membranes lipidiques constituent pour le biologiste ou le biophysicien l'élément de base de la paroi cellulaire $[1$,

Finalement, pour le physicien, ce sont des objets modèles mous, à quasi-deux dimensions, qui sont soumis à l'agitation thermique du solvant. Ces systèmes possèdent donc des propriétés physiques remarquables mettant en jeu une gamme d'échelles spatiales très large : échelle moléculaire nanométrique pour l'épaisseur de la membrane, de la centaine de nanomètres à la dizaine micron pour la taille des vésicules, jusqu'au centimètre pour les membranes supportées.

Les membranes de tensioactifs se retrouvent dans différents systèmes : phases lamellaires [3], vésicules [4], membranes supportées [5]. De nombreuses études ont porté sur leur caractérisation structurale, essentiellement par diffusion de rayons X ou de neutrons. Dans le cas des membranes phospholipidiques on peut trouver une très bonne revue des études structurales par J. F. Nagle [3]. L'étude des fluctuations thermiques a été abordée dans de nombreux travaux, tant sur le plan expérimental que théorique. D'un point de vue théorique, les travaux pionniers de Helfrich [6] concernent essentiellement les membranes planes. Par la suite, différents auteurs se sont intéressés aux fluctuations de vésicules [4]. Les fluctuations des membranes sont généralement gouvernées par plusieurs paramètres :

- le module de courbure intrinsèque de la membrane $\kappa$ qui stabilise les fluctuations de petites longueurs d'onde ;

- pour les membranes non isolées, le potentiel $U$ (par unité de surface) d'interaction avec un substrat (membrane supportée, vésicules sur une surface) ou avec une autre membrane qui contrôle les fluctuations à grandes longueurs d'onde ;

- la tension de surface $\gamma$, qui dans certains cas domine les échelles de longueurs intermédiaires ;

${ }^{*}$ Université Louis Pasteur 
De nombreuses études expérimentales ont été menées pour étudier les fluctuations des membranes, sur des phases lamellaires [3, 7, 8] et sur des vésicules [9-17] en utilisant des techniques variées.

Dans ce cours d'illustration, après avoir donné une introduction générale aux systèmes moléculaires organisés et plus particulièrement aux propriétés des membranes (partie II), nous nous concentrerons uniquement sur les études à partir de diffusion de rayonnement au travers de trois exemples :

- L'étude des fluctuations de phases multilamellaires de membranes de tensio-actifs par diffusion de rayons $\mathrm{X}[7,18]$ constitue la première mise en évidence expérimentale quantitative de la répulsion de Helfrich. Nous décrirons ici les travaux de Safinya et al. sur le système quaternaire SDS/eau/ pentanole/dodécane (partie III).

- L'étude indirecte par réflectivité de neutrons des fluctuations de membranes supportées à partir du gonflement observé à la transition gel/fluide [19] (partie IV B) ;

- L'étude directe du spectre de fluctuations d'une membrane unique par diffusion de rayons $X$ $[20,21]$ (partie IV C) dans un régime submicronique.

\section{MOLÉCULES AMPHIPHILES-SYSTÈMES AUTO-ASSEMBLÉS}

\subsection{Molécules amphiphiles}

Les molécules amphiphiles présentent un caractère double, avec une partie polaire présentant une grande affinité pour les solvants polaires (comme l'eau) et une partie aliphatique hydrophobe. Elles sont donc à la fois hydrophiles et lipophiles. Il existe une grande variété de molécules amphiphiles : les acides gras, les phospholipides, des copolymères à blocs... La partie hydrophobe est généralement constituée d'une ou de plusieurs longues chaînes carbonées. La partie hydrophile peut être chargée négativement (acide gras, sulfate, phosphatidylsérine...), positivement (CTAB...), neutre ou zwitterionique (Figure 2).

La double affinité des molécules amphiphiles leur confère des propriétés remarquables. Leur caractère tensioactif décrit la capacité de ces molécules à stabiliser les interfaces entre eux solvants non miscibles. Les films et les bulles de savons sont une illustration bien connue de cette propriété, la stabilisation du film d'eau pouvant conduire à des films spectaculaires de grande taille (Figure 1).

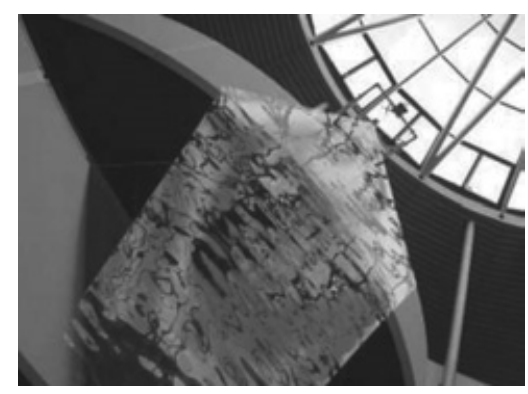

Figure 1. Film de savon géant $(10 \mathrm{~m} \times 3 \mathrm{~m})$ (d'aprés P. Ballet, F. Graner [22].

Les propriétés auto-associatives expliquent la formation de structures moléculaires complexes à l'échelle mésoscopique. En solution, les tensio-actifs s'auto-assemblent pour former des structures d'achitectures variées, stables ou métastables d'un point de vue thermodynamique. Les structures obtenues tendent à protéger les parties hydrophobes ou hydrophiles du solvant suivant la nature polaire ou apolaire de celui-ci.

On distingue généralement un premier niveau d'organisation : micelle sphérique, micelle cylindrique (vermicelle), bicouche. Ces objets peuvent présenter un second niveau d'organisation : phase hexagonale, lamellaire, cubique, éponge, vésicules de morphologies et topologies variées... 

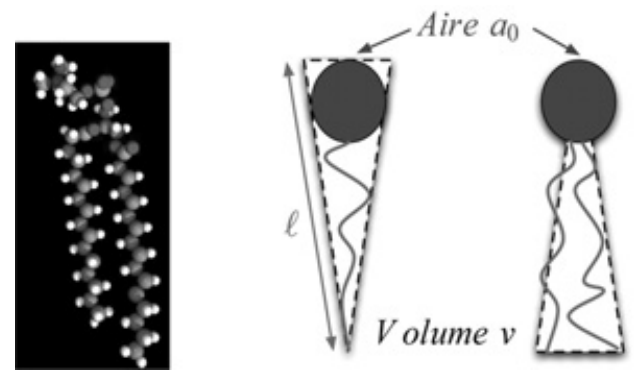

Figure 2. Exemple de molécule amphiphile (un phospholipide, ici une molécule de DPPC) et définition des paramétres géométriques $v, a_{0}$ et $\ell$.

Il est possible de décrire de façon qualitative les propriétés auto-associatives des molécules amphiphiles à partir de considérations géométriques simples.

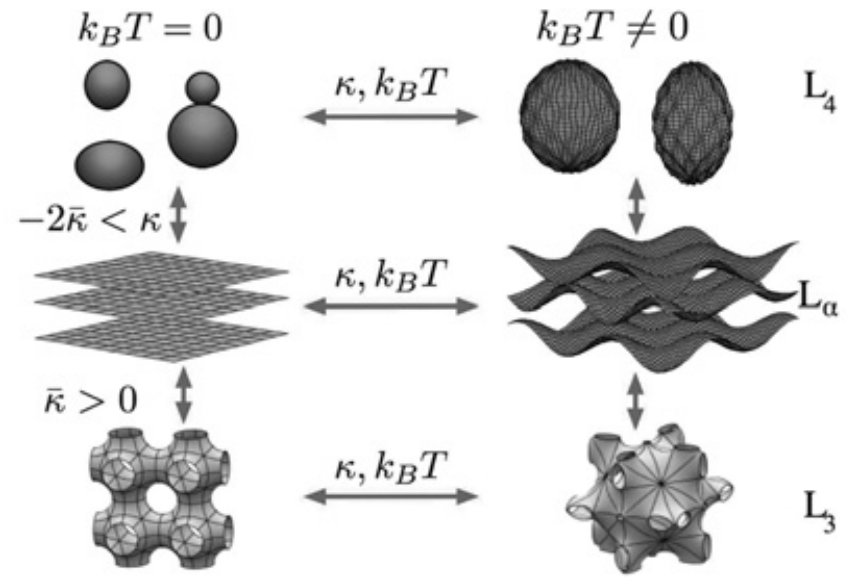

Figure 3. Diagramme de phases schématique pour les systémes lamellaires.

Pour cela, on définit la surface moyenne $a_{0}$ occupée par la tête d'une molécule, la longueur de la chaîne $\ell$ et le volume moyen de la molécule $v$ (Figure 2). Le volume $V$ et la surface $S$ de la structure formée par l'association des molécules amphiphiles (micelles sphériques, micelles cylindriques, lamelles) sont donnés par $V=\mathcal{N} v$ et $\mathcal{S}=\mathcal{N}\left(a_{0}\right.$. Le paramétre $V /(\ell S)$, caractéristique de la géométrie de la structure, est indépendant du nombre de molécules et égal au volume réduit $v /\left(a_{0} \ell\right)$. Les propriétés géométriques des molécules amphiphiles déterminent donc celles de la structure complexe formée par association en solution :

- si $v /\left(a_{0} \ell\right)<1 / 3$, on obtient une phase de type micellaire sphérique.

- si $1 / 3<v /\left(a_{0} \ell\right)<1 / 2$, la phase est de type micellaire cylindrique.

- si $1 / 2<v /\left(a_{0} \ell\right)$, la phase est de type lamellaire.

Les phases micellaires peuvent présenter une grande richesse de structures suivant que le système est plus ou moins dilué. Il existe toute une classification de ces phases [23-25] qui ne sera pas abordée ici.

Nous allons nous intéresser uniquement aux phases formées à partir de bicouches. On peut distinguer la phase lamellaire ordonnée $L_{\alpha}$, les phases lamellaires multiconnectées ordonnées (phase cubique) ou désordonnées (phase éponge $L_{3}$ ) et les phases de vésicules $L_{4}$ (Figure 3). 


\subsection{Généralités sur la physique des membranes}

Énergie de courbure à température nulle

L'étude théorique des membranes a débuté avec les travaux de Canham puis ceux de Helfrich [26, 27]. A l'échelle macroscopique, ces derniers ont proposé de les décrire à partir d'une énergie élastique de courbure phénoménologique basée sur les propriétés des films minces :

$$
\mathcal{H}=\frac{1}{2} \kappa \int d S\left(C_{1}+C_{2}-C_{0}\right)^{2}+\bar{\kappa} \int d S C_{1} C_{2}+\gamma \int d S
$$

La forme de la membrane est ici décrite par les deux courbures principales $C_{1}$ et $C_{2}\left(C_{i}=R_{i}^{-1}\right)$ (Figure 4). L'énergie (équation 1) peut-être vue comme un développement en puissance des invariants du tenseur de courbure. Le dernier terme de cette équation est un terme de tension de surface. $C_{0}$ est la courbure spontanée de la membrane qui peut-être associée à une asymétrie de composition de la bicouche. Le paramètre $\kappa$ s'appelle constante (ou module) élastique de courbure moyenne. Il est possible de le mesurer expérimentalement par des méthodes d'aspiration par micropipette, ou par l'étude de l'amplitude des fluctuations thermiques de la membrane $[12,13]$. On obtient des valeurs de l'ordre de $10-50 k_{B} T$ pour les membranes phospholipidiques. Le paramètre $\bar{\kappa}$ est appelé constante ou module élastique de courbure gaussienne et est associé à la topologie de la membrane. Le théorème de GaussBonnet [28] permet d'évaluer l'intégrale de la courbure gaussienne pour une surface fermée sans bords. Il montre que cette intégrale dépend uniquement de la topologie de la surface. Il s'exprime sous la forme suivante :

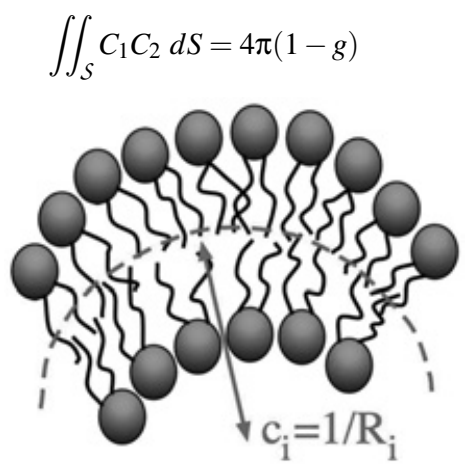

Figure 4. Definition des rayons de courbures d'une membrane.

La constante $g$ est appelée genre topologique et correspond au nombre de «poignées » de la surface ( $g=0$ pour la sphère, $g=1$ pour le tore). Pour un système constitué de plusieurs surfaces fermées, on peut écrire la contribution de la courbure gaussienne à l'énergie de courbure sous la forme :

$$
\bar{\kappa} \iint_{\mathcal{S}} C_{1} C_{2} d S=4 \pi \bar{\kappa}\left(N-N_{h}\right)
$$

où $N$ est le nombre de composantes déconnectées et $N_{h}$ le nombre total de poignées.

Cette contribution à l'énergie de courbure agit comme un potentiel chimique qui gouverne la topologie du système. En particulier, il est important de connaitre le signe de $\bar{\kappa}$. C'est lui qui contrôle l'apparition de « poignées » sur la surface, c'est-à-dire la formation de point selle de courbure moyenne quasi-nulle et de courbure gaussienne négative.

Il est intéressant de noter que l'énergie de courbure est invariante d'échelle. Il n'existe donc pas de longueur caractéristique dans ce problème. Nous verrons plus loin que cette invariance est brisée si on prend en compte la renormalisation des constantes élastiques par les fluctuations thermiques de la membrane. 


\section{Stabilité des phases lamellaires}

Nous avons vu qu'une bicouche phospholipidique symétrique peut être décrite par une énergie de courbure du type Helfrich :

$$
\varepsilon_{e l}=\frac{1}{2} \kappa \iint_{\mathcal{S}}\left(\frac{1}{R_{1}}+\frac{1}{R_{2}}\right)^{2} d S+4 \pi \bar{\kappa}(N-N h)
$$

Il est possible, à partir de cette énergie, d'étudier la stabilité d'une phase lamellaire. Nous avons vu que le terme de courbure gaussienne est celui qui contrôle la topologie du système alors que les fluctuations de forme de la surface sont gouvernées par la courbure moyenne. On peut imaginer deux mécanismes de déstabilisation de la membrane :

- par la création d'un passage. On montre facilement que le passage coûte une énergie $-4 \pi \bar{\kappa}$ (en première approximation le passage est une surface minimale de courbure moyenne nulle). On gagne donc de l'énergie à créer des passages si $\bar{\kappa}>0$.

- par la formation d'une vésicule. L'énergie de courbure d'une vésicule sphérique se met sous la forme $4 \pi(2 \kappa+\bar{\kappa})$. On gagne donc de l'énergie à créer des vésicules si $\bar{\kappa}<-2 \kappa$.

On peut donc conclure que la phase lamellaire $L_{\alpha}$ est stable pour $-2 \kappa<\bar{\kappa}<0$.

Pour $\bar{\kappa}>0$ il se forme une phase multiconnectée ordonnée (du type phase cubique) ou désordonnée (phase éponge) si les fluctuations thermiques sont importantes. Si $\bar{\kappa}<-2 \kappa$ il se forme une phase de vésicules dont la forme et les fluctuations dépendent de la valeur de $\kappa$ et de la contrainte de volume.

L'ensemble de ces propriétés est résumé sur la Figure 3.

Effet de la température : renormalisation des constantes élastiques

L'énergie élastique de courbure introduite précédemment décrit le comportement de la membrane à température nulle. Lorsqu' on cherche à tenir compte de la température, on doit prendre en compte les fluctuations thermiques de la membrane. La théorie de la renormalisation montre alors que l'on doit remplacer la membrane réelle par une membrane effective lissée dont les caractéristiques dépendent de l'échelle $\xi$ d'observation.

La renormalisation des constantes élastiques de courbures moyenne et gaussienne [29-32] conduit à définir une longueur de persistance caractéristique de la bicouche et fonction de la température :

$$
\xi_{K}=a \exp \left[\frac{4 \pi \kappa}{3 k_{b} T}\right]
$$

Pour des échelles d'observation inférieures à $\xi_{K}$, la membrane apparaît comme tendue, alors qu'à des échelles supérieures à $\xi_{K}$, les fluctuations deviennent très importantes. On peut donner quelques ordres de grandeurs de $\xi_{K}$ (avec par exemple $a=20 \AA$ ) :

- pour $\kappa \simeq 10 k_{B} T$ classique pour une bicouche fluide de phospholipides, on obtient $\xi_{K}=10^{19} \mathrm{~m}$ ! Cette valeur est énorme devant la taille des systèmes étudiés. Dans ce cas, la membrane est lisse à toutes les échelles et on peut négliger les fluctuations.

- pour $\kappa \simeq k_{B} T$, on obtient $\xi_{K} \simeq 1 \mu m$. Dans ces conditions, on peut observer des fluctuations importantes de la membrane pour des échelles de l'ordre du micron. Ces fluctuations sont observables sur des vésicules géantes [33], ou des systèmes biologiques [12].

- dans le cadre des microémulsions, on peut avoir $\kappa \simeq k_{B} T / 10$. Les fluctuations sont alors très importantes. La taille caractéristique de la microémulsion est fixée par $\xi_{K}$.

Le modèle présenté ci-dessus ne prend pas en compte l'effet de la dilution du système [34]. On obtient donc un diagramme de phase indépendant de la concentration en lipides. 
Fluctuations thermiques autour de l'équilibre

Sous l'effet de l'agitation thermique, la membrane fluctue autour de sa position d'équilibre. De nombreuses études [4] se sont attachées à décrire l'effet de ces fluctuations sur la forme des vésicules. Nous nous limiterons ici au cas de membranes quasi-planes. Dans ces conditions, on peut décrire la forme de la surface dans la représentation de Monge où la position d'un point de la membrane est repérée par sa hauteur $u(\vec{r})=u(x, y)$ par rapport à un plan de référence parallèle à la position moyenne de la membrane (Figure 5).

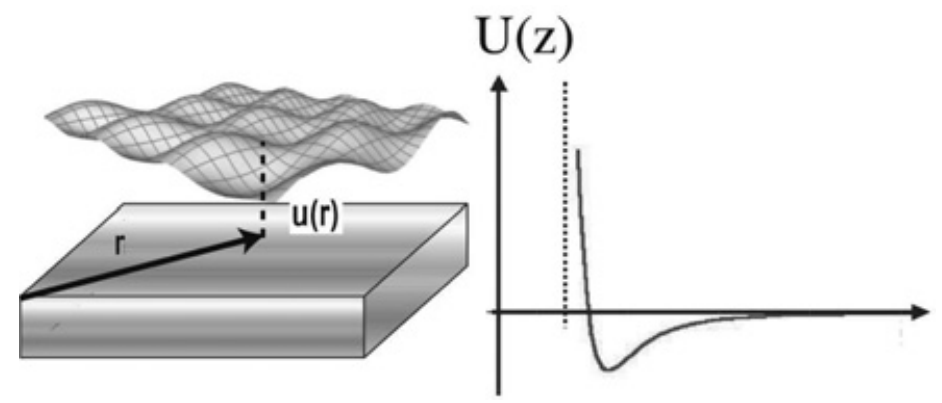

Figure 5. Représentation de Monge.

L'énergie de la membrane peut alors être linéarisée et s'exprime comme :

$$
\mathcal{H} \simeq \frac{1}{2} \kappa \int d x d y(\Delta u)^{2}+\gamma \int d x d y(\vec{\nabla} u)^{2}+\int d x d y U(u(\vec{r}))
$$

On a ajouté ici un potentiel $U(u(\vec{r}))$ qui décrit les interactions entre la membrane et un substrat solide ou une autre membrane.

Si on linéarise le potentiel autour de l'équilibre, l'énergie est quadratique en la déformation $u(\vec{r})$. On peut donc décomposer la déformation de la membrane en modes de Fourier d'amplitude $u_{q}(u(\vec{r})=$ $\left.1 /(2 \pi)^{2} \oint d^{2} \vec{r} u(\vec{r}) e^{i \vec{q} \cdot \vec{r}}\right)$ et le théorème d'équipartition de l'énergie permet d'écrire l'amplitude moyenne carrée de chaque mode comme :

$$
\left\langle\left|u_{q}\right|^{2}\right\rangle=\frac{k_{B} T}{\widetilde{U}^{\prime \prime}+\gamma q^{2}+\kappa q^{4}}
$$

\section{Interaction de Helfrich}

Les fluctuations de forme d'une membrane lorsqu'elle se trouve au voisinage d'un substrat solide (mur dur) ou d'une autre membrane conduisent à une interaction d'origine entropique qui dépend de la rigidité des membranes et de la température. Cette interaction a été décrite pour la première fois par W. Helfrich en 1978 [6]. Elle permet en particulier de comprendre la remarquable stabilité des phases lamellaires de tensioactifs neutres.

Considérons simplement une membrane unique de module de courbure $\kappa$, en négligeant dans un premier temps la tension de surface. Les fluctuations de cette membrane sont confinées entre deux murs durs distants de $d$ (Figure 6(a)). On suppose qu'il n'existe que des interactions stériques à très courte portée entre les murs et la membrane. L'amplitude caractéristique des fluctuations de la membrane est limitée par la présence des murs. On peut raisonnablement supposer qu'un choc de la membrane sur un mur décorréle complètement les fluctuations. On voit alors la membrane comme une mosaïque de patch 


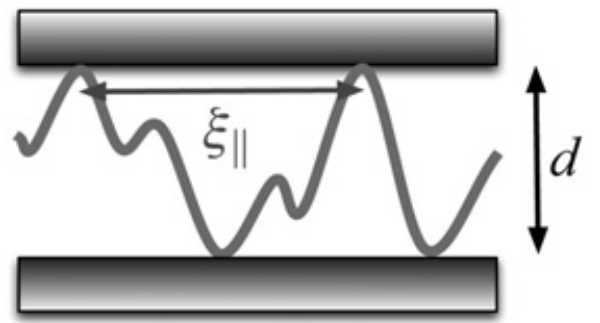

(a)

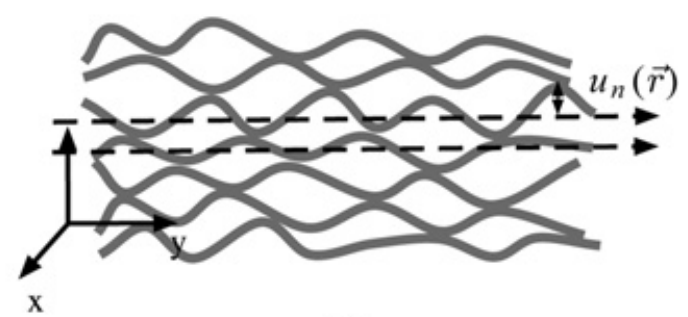

(b)

Figure 6. Géométries symétriques décrites théoriquement par Helfrich [6] : (a) membrane confinée entre deux murs ; (b) membrane confinée dans une phase multilamellaire.

indépendants de taille $\xi_{\|}^{2}$ et d'amplitude de fluctuation moyenne $d^{2}$. On peut maintenant relier ces deux grandeurs en utilisant l'expression du spectre de fluctuations (équation 7) avec $\widetilde{U}^{\prime \prime}=0$ et $\gamma=0$ :

$$
\begin{aligned}
\left\langle\left|u_{q}\right|^{2}\right\rangle & =\frac{k_{B} T}{\kappa q^{4}} \\
\Rightarrow d^{2} & \sim \int d^{2} \vec{q}\left\langle\left|u_{q}\right|^{2}\right\rangle \sim \frac{k_{B} T}{\kappa} \xi_{\|}^{2}
\end{aligned}
$$

Il reste à calculer la variation d'énergie libre $\Delta \mathcal{F}_{S}$ par unité de surface due au confinement de la membrane. La courbure de la membrane liée à la présence des murs est simplement donnée par $R^{-1} \sim d / \xi_{\|}^{2}$. On en déduit la contribution enthalpique :

$$
\Delta \varepsilon_{c} \simeq \kappa\left(\frac{d}{\xi_{\|}^{2}}\right)^{2}
$$

Les bouts de membrane de taille $\xi_{\|}^{2}$ étant indépendants, le confinement réduit leur entropie de $k_{B}$ et on obtient :

$$
\Delta \mathcal{S}_{c} \simeq \frac{k_{B} T}{\xi_{\|}^{2}}
$$

On obtient donc la variation d'énergie libre due au confinement qui décrit les interactions entre la membrane et les murs :

$$
\Delta \mathcal{F}_{\text {Helfrich }} \propto \Delta \mathcal{E}_{c}-T \Delta S \propto \frac{\left(k_{B} T\right)^{2}}{\kappa} \frac{1}{d^{2}}
$$

C'est une interaction répulsive, proportionnelle à l'énergie d'agitation thermique $k_{B} T$ et inversement proportionnelle au module de courbure de la membrane. On introduit généralement une constante $c_{H}$ et on note l'énergie de répulsion de Helfrich :

$$
V_{H e l f} \simeq c_{H} \frac{\left(k_{B} T\right)^{2}}{\kappa} \frac{1}{d^{2}}
$$

De nombreuses études théoriques et numériques ont développé les travaux pionniers de Helfrich. U. Seifert [35] a proposé une généralisation de l'interaction de Helfrich au cas de membranes sous tension $(\gamma \neq 0)$. 
Dans le cas asymétrique d'une membrane neutre fluctuant près d'un seul substrat (ou d'une seule autre membrane), l'interaction entropique (répulsion de Helfrich) repousse la membrane qui peut décoller. Dans cette situation, la membrane interagit via un potentiel d'origine microscopique [36] :

$$
\begin{aligned}
& U(z)=U_{\text {hydratation }}(z)+U_{v d W}(z) \\
& U(z)=P_{h} l_{h y} e^{-z / l_{H_{2}} O}-\frac{H}{12 \pi}\left[\frac{1}{z^{2}}-\frac{1}{\left(z+\delta^{2}\right)}\right]
\end{aligned}
$$

où $U_{\text {hydratation }}$ est l'interaction d'hydratation qui décrit la répulsion des têtes lipidiques hydratées [36] et $U_{v d W}$ est le potentiel de van der Waals. Pour des membranes lipidiques, $\mathrm{H}$ est typiquement de l'ordre de $10^{-20}-10^{-21} \mathrm{~J}, P_{h} \sim 10^{8}-10^{9}$ Pa et $l_{h y} \sim 0.2-0.3 \mathrm{~nm}$. Leibler et al. [37] ont étudié le cas asymétrique d'une membrane interagissant avec un mur (ou une membrane) dans la limite des grandes fluctuations, en utilisant les concepts du groupe de renormalisation. Le cas asymétrique dans le régime des petites fluctuations a été abordé de façon autocohérente par Mecke et al. [38].

Dans le cas de tensioactifs neutres, en ne prenant en compte que des interactions de van der Waals, on peut montrer que la position et les fluctuations de la membrane sont contrôlées par le paramètre sans dimension $\beta=\left(k_{B} T\right)^{2} /(H \kappa)$ :

- si $\beta$ est petit, la membrane fluctue doucement dans le minimum du potentiel microscopique (équation 14);

- lorsque $\beta$ augmente l'amplitude des fluctuations augmente et la membrane est repoussée par le substrat [38] ;

- lorsque $\beta$ atteint une valeur critique $\beta_{u} \sim 0.2-0.3$ la membrane décolle complètement du substrat (transition de décollement [36, 37]).

\section{3. ÉTUDE EXPÉRIMENTALES DES FLUCTUATIONS DE SYSTÈMES MULTILAMELLAIRES}

\subsection{Membrane de Dodécyl Sulfate de Sodium (SDS)}

\section{Le système quaternaire : SDS, pentanol, eau, dodécane}

Safinya et al. [7] ont étudié les fluctuations de membranes par diffusion de rayons $\mathrm{X}$ sur un système quaternaire ( $\mathrm{SDS}=\mathrm{C}_{12} \mathrm{H}_{25} \mathrm{OSO}_{3} \mathrm{Na}$, pentanol, eau, dodécane). Dans ce système le SDS est le tensioactif et le pentanol joue le rôle de cotensioactif. La Figure 7 montre une coupe du diagramme de phase pour un rapport en masse eau/SDS constant [39] où l'on peut repérer différentes phases dont une phase lamellaire inverse notée $L_{\alpha}$.

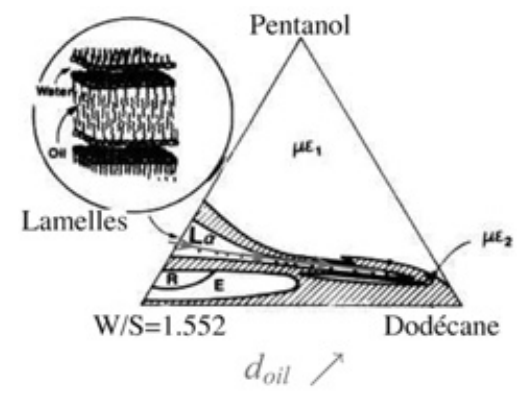

Figure 7. Coupe du diagramme de phase du systéme quaternaire SDS/pentanol/eau/doddécane pour un rapport en masse eau/SDS=1.552 [39]; $\mu \in_{1}$ et $\mu \in_{2}$ sont deux phases de microémulsion, (E) et (R) les phases cristallines hexagonale et rectangulaire et $L_{\alpha}$ la phase lamellaire. 
Cette phase lamellaire inverse est constituée de films d'eau d'épaisseur $d_{w}$ stabilisés par le SDS, périodiquement répartis dans une solution de dodécane (périodicité $\mathrm{d}=$ pas smectique, $d_{o}=d-d_{w}$ est l'épaisseur du film d'huile). Les lamelles d'eau sont neutres et il n'y a donc pas d'interaction électrostatique entre les membranes. D'autre part le pentanol s'insère dans la membrane et réduit fortement le module de courbure qui devient de l'ordre de $k_{B} T$. Finalement la variation de la fraction en masse de dodécane $x$ permet de faire varier le pas smectique d entre 20 et $200 \mathrm{~nm}$ en conservant une épaisseur de lamelle pratiquement constante.

\section{Diffusion de rayonnement-Loi de Caillé}

La diffusion statique de rayonnement (neutrons ou rayons $X$ ) aux petits angles se prête particulièrement bien à l'étude des systèmes moléculaires organisés comme les phases lamellaires. Le système est éclairé avec un rayonnement incident de direction $\vec{u}_{i}$ (vecteur d'onde $\vec{k}_{i}=2 \pi / \lambda \times \vec{u}_{i}$ ) et on détecte l'intensité de l'onde diffusée dans la direction $\vec{u}_{d}$ (vecteur d'onde $\vec{k}_{d}=2 \pi / \lambda \times \vec{u}_{d}$ ). On définit alors le vecteur d'onde de diffusion $\vec{q}=\vec{k}_{d}-\vec{k}_{i}\left(|\vec{q}|=4 \pi / \lambda \times \sin (\theta / 2)\right.$ où $\theta=\left(\vec{u}_{i}, \vec{u}_{d}\right)$ est l'angle de diffusion). L'intensité diffusée par le système est reliée à la densité de centres diffuseurs $\rho(\vec{r})$ :

$$
I(\vec{q})=\left\langle\left|\int_{\mathcal{V}} \rho(\vec{r}) e^{i \vec{q} \cdot \vec{r}} d^{3} \vec{r}\right|^{2}\right\rangle
$$

On peut décomposer cette intensité en deux termes :

$$
I(\vec{q}) \propto \frac{P(q) S(q)}{q^{2}}
$$

où $P(\vec{q})$ est le facteur de forme qui est associé à la structure d'une membrane et $S(\vec{q})$ le facteur de structure qui décrit la disposition des membranes dans l'échantillon. Dans la suite on va se concentrer sur le facteur de structure.

Dans une approche très simplifiée, pour un système de $\mathcal{N}$ membranes identiques périodiquement réparties avec un pas smectique $\mathrm{d}$, on s'attend à des pics de diffraction pour des vecteurs de transfert $q_{z}=q_{m}=2 \pi / d \times m$. La situation expérimentale n'est pas aussi simple. Le système est généralement constitué d'un ensemble de domaines lamellaires d'orientations variées (on parle parfois de poudre par analogie avec la cristallographie) $[40,41]$ ce qui complique l'analyse. Il faut aussi prendre en compte le facteur de forme de la membrane. Finalement, les fluctuations de position des membranes ont un effet dramatique sur les pics de diffraction en détruisant l'ordre à longue portée dans le système. Cet effet spécifique à la dimensionnalité du système est connu sous le nom d'instabilité de Landau-Peierls [42].

Pour comprendre l'effet des fluctuations de position sur la figure de diffraction, on doit calculer la fonction de corrélation des hauteurs des membranes [43,44]. On considère alors un système multilamellaire de $\mathcal{N}$ membranes identiques, quasi-planes, de module de courbure $\kappa$. Sous l'effet de l'agitation thermique, celles-ci fluctuent autour de leur position moyenne. On décrit le système en prenant en compte l'énergie de courbure et une interaction quadratique entre les membranes :

$$
\mathcal{H}=\frac{1}{2} \kappa \sum_{n=1}^{\mathcal{N}} \int d^{2} \vec{r}_{\|}\left(\Delta u_{n}\right)^{2}+\frac{1}{2} B \sum_{n=1}^{\mathcal{N}} \int d^{2} \vec{r}_{\|}\left[u_{n+1}\left(r_{\|}\right)-u_{n}\left(r_{\|}\right)\right]^{2}
$$

ou $\kappa$ est le module de courbure d'une membrane et $u_{n}(\vec{r})$ l'écart de position de la $\mathrm{n}^{\text {ième }}$ membrane par rapport à son plan moyen. $B$ est la raideur de l'interaction entre les membranes et correspond à la compressibilité du système. En notant $d$ le pas smectique du système, on peut passer maintenant à la limite continue et écrire :

$$
\mathcal{H}=\int d z \int d^{2} \vec{r}_{\|}\left[\frac{1}{2} K(\Delta u)^{2}+\frac{1}{2} B\left(\frac{\partial u}{\partial z}\right)^{2}\right]
$$


où on a défini un module de courbure par unité de longueur $K=\kappa / d$. Cette énergie est à nouveau quadratique. Les modes de Fourier sont donc découplés. On doit distinguer les variations selon $\mathrm{z}\left(q_{z}\right)$ des variations dans le plan des membranes $\left.\left(q_{\|}=\left(q_{x}, q_{y}\right)\right)\right)$ :

$$
\left\langle\left|u_{q}\right|^{2}\right\rangle=\frac{k_{B} T}{B q_{z}^{2}+K q_{\|}^{4}}
$$

On constate que l'amplitude de fluctuation d'un mode peut diverger quelle que soit la température si on fait tendre la longueur d'onde de la déformation vers l'infini $(\vec{q} \rightarrow \overrightarrow{0})$ et que $\left\langle|u|^{2}\right\rangle \propto \log \left[\frac{L}{\delta}\right]$ diverge doucement avec la taille du système. On parle d'ordre à quasi-longue portée. On montre alors [43, 44] que le facteur de structure qui donne l'intensité diffusée prend l'allure suivante :

$$
\begin{gathered}
S\left(0,0, q_{z}\right) \sim\left|q_{z}-q_{m}\right|^{-2+\eta_{m}} \\
S\left(q_{\perp}, 0, q_{m}\right) \sim q_{\perp}^{-4+2 \eta_{m}}
\end{gathered}
$$

avec $\eta_{m}=k_{B} T q_{m}^{2} / 8 \pi \sqrt{B K}[43]$.

La conséquence de la disparition de l'ordre à longue-portée dans le système est que les pics de Bragg $\left(q_{m}=2 \pi / d m\right)$ se transforment en singularités en loi de puissance qui décroissent très rapidement.

En l'absence d'interaction électrostatique, la compressibilité B est dominée par la répulsion de Helfrich. On peut calculer $\mathrm{B}$ de façon autocohérente à partir de l'énergie libre du système (qui dépend elle-même de B) [6] :

$$
\begin{aligned}
B=d \frac{\partial^{2} \mathcal{F}}{\partial d^{2}} & =\frac{\left(k_{B} T\right)^{2}}{\kappa} \frac{1}{d^{4}} \\
B K & =\frac{9 \pi^{2}}{64} \frac{\left(k_{B} T\right)^{2}}{d^{4}}
\end{aligned}
$$

On en déduit donc l'expression des exposants de Caillé :

$$
\eta_{m}=\frac{4}{3} m^{2}
$$

qui devient, lorsque l'on prend en compte l'épaisseur de la membrane :

$$
\eta_{m}=\frac{4}{3}\left(1-\frac{\delta}{d}\right)^{2} m^{2}
$$

Safinya et al. [7] ont étudié en détails la diffusion de rayons $\mathrm{X}$ par une phase lamellaire. On résume ici l'essentiel de leurs résultats. La Figure 8 montre l'évolution du premier pic en fonction de la fraction en masse de dodécane $\mathrm{x}$, donc pour différentes valeurs du pas smectique. On vérifie tout d'abord que la position du premier pic $q_{0}=2 \pi / d$ se déplace vers les petits vecteurs d'ondes lorsque la fraction en masse de dodécane augmente. On observe aussi un élargissement des pics lorsque le pas smectique d (ou $\mathrm{x}$ ) augmente, ce qui indique une augmentation du désordre thermique.

Les courbes tracées sur la Figure 8 sont des ajustements des données expérimentales de rayons $\mathrm{X}$ avec une loi de puissance du type de l'équation 21. Ces expériences permettent donc la détermination simultanée du pas smectique (via la position du pic $q_{0}$ ) et de l'exposant de Caillé $\eta$ (relié à la largeur du pic). Elles permettent donc de tester directement l'expression (24) du paramètre de Caillé et constituent la première mise en évidence expérimentale de l'interaction de Helfrich. 


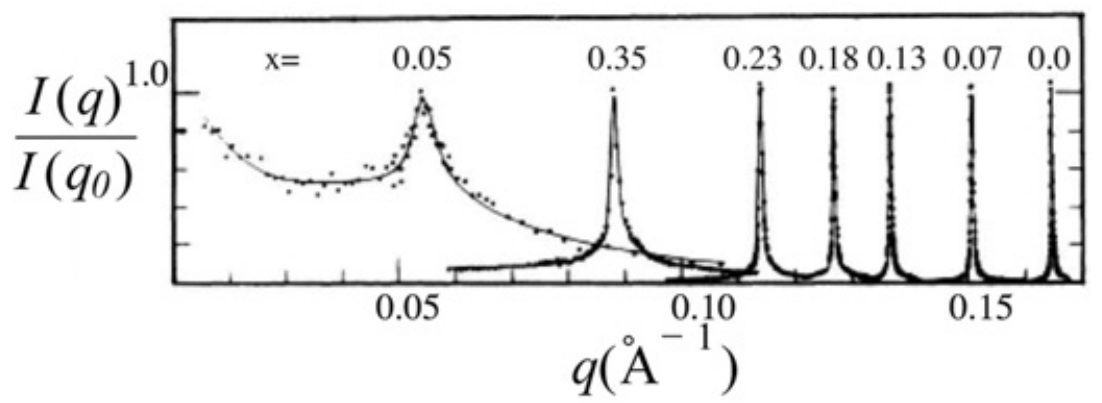

Figure 8. Evolution du premier pic de Bragg en fontion en masse de dodécane $x$ (d'aprés [7]).

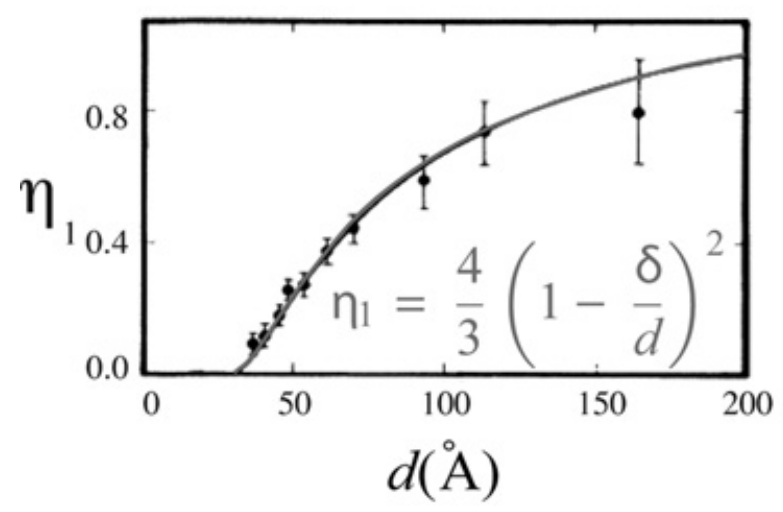

Figure 9. Variation du paramétre de Caillé $\eta_{1}$ en fontion du pas smectique d : $(\bullet)$ points expérimentaux ; la courbe correspond à un adjustement des données à partir de 1'equation (24).

\subsection{Multicouches de phospholipides}

Le cas des multicouches phospholipidiques est plus complexe. Les modules de courbure sont beaucoup plus élevés que dans le cas précédent $\left(\kappa \sim 20-50 k_{B} T\right.$ en phase fluide). Les expériences de diffusion sont donc paradoxalement limitées à très peu d'ordres de diffraction [3]. De plus, il est difficile de mesurer indépendamment le module de courbure $\kappa$ et la compressibilité $B$ [45]. L'analyse des expériences de diffusion de rayonnement (neutrons ou rayons $\mathrm{X}$ ) est par conséquent extrêmement complexe [3].

Différentes approches ont été esquissées pour essayer de simplifier cette analyse. Vogel et al. [46] ont développé des études sur des systèmes multilamellaires très orientés (dépôt par spin-coating) qui permettent de s'affranchir de la perte d'ordre à longue portée en imposant des conditions aux limites sur le substrat. Les mêmes auteurs [47] ont aussi développé des études de diffusion hors-spéculaire de neutrons sur ce même type de systèmes. Nous ne développerons pas ici ces études mais nous allons maintenant nous concentrer sur une autre approche qui consiste à étudier les fluctuations d'une membrane « unique ». 


\section{4. ÉTUDE DES FLUCTUATIONS D'UNE MEMBRANE UNIQUE}

\subsection{Introduction}

\section{Contexte}

Étudier les fluctuations d'une membrane phospholipidique unique peut se faire de différentes fa̧ons suivant les échelles spatiales que l'on souhaite explorer. Beaucoup d'études ont été réalisées avec des techniques optiques. La première mise en évidence des fluctuations d'une membrane phospholipidique est due à Brochard et Lennon [12] à partir de l'observation du scintillement des globules rouges.

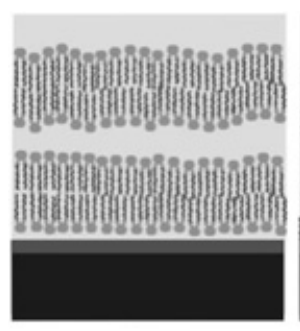

(a)

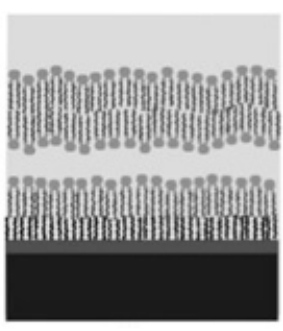

(b)

Figure 10. (a) Double bicouches supportées [51] et (b) systéme mixte OTS-monocouche greffé bicouche supportée [50].

D'autres techniques optiques permettent d'avoir accès à certaines informations sur les fluctuations des membranes. La microscopie à contraste interférentiel en réflexion (RICM) permet par exemple d'étudier l'adhésion de vésicules sur des substrats [48]. On peut aussi essayer d'analyser le contour de la vésicule. Cette technique a permis à Pécréaux et al. [13] de mesurer le spectre de fluctuations de bicouches dans le domaine submicronique $\left(0.5 \cdot 10^{6} \mathrm{~m}^{-1}<q<10^{5} \mathrm{~m}^{-1}\right)$.

Finalement, on peut citer les études basées sur les travaux de Evans et al. [13] qui consistent à mesurer la surface cachée dans les fluctuations de la membrane d'une vésicule en aspirant celle-ci dans une micropipette.

\section{Doubles bicouches : une membrane supportée quasi-libre}

On appelle généralement membranes supportées les systèmes où une bicouche de phospholipides est déposée sur un substrat solide, généralement hydrophile[5]. Ils ont de nombreuses applications [5]. Est-il possible de les utiliser pour étudier les fluctuations d'une membrane « unique » avec les techniques de diffusion de rayonnement? En dehors des considérations expérimentales sur la possibilité de mesurer un signal diffusé sur des systèmes aussi minces (épaisseur $\sim 1 \mathrm{~nm}$ ), leur principal inconvénient vient de leur forte interaction avec le substrat qui réduit de façon drastique leurs fluctuations. Plusieurs solutions [49-51] ont été proposées pour obtenir une membrane supportée plus libre. Le principe est toujours le même : introduire un « espaceur » entre la bicouche et le substrat. Dans ce cour, nous nous concentrerons sur deux approches : la première consiste à déposer deux membranes sur le susbtrat (la première bicouche joue le rôle d'espaceur et la seconde est plus libre de fluctuer [51]); la seconde [50] utilise un système mixte OTS-Lipide qui permet d'avoir un « espaceur » plus lié au substrat. Ces deux systèmes sont représentés schématiquement sur la Figure 10.

Ils présentent de nombreux avantages. La membrane quasi libre est accessible et non-confinée, sa position est bien contrôlée. On peut envisager d'étudier ses propriétés dynamiques dans des conditions proches d'une membrane « unique ». En revanche, ces systèmes sont très fragiles et difficiles à réaliser. 
L'interaction relativement faible de la seconde membrane avec le substrat complique le dépôt des couches de lipides. On est amené à utiliser une combinaison de techniques de déposition de type LangmuirBlodgett et Langmuir-Schaefer.

La première étape dans l'étude de ces systèmes a consisté à caractériser de façon précise leur structure. Cela a été essentiellement réalisé en utilisant la réflectivité de neutrons [50,51]. Le détail de ces caractérisations est décrit dans le cours de G. Fragneto. On rappellera ici simplement que cette technique permet de remonter au profil de longueur de diffusion neutronique normal à la surface de l'échantillon. L'ajustement des courbes expérimentales est délicat, essentiellement car on perd toutes les informations relatives à la phase du profil de longueur de diffusion. On procède donc à une caractérisation par étape, en déterminant successivement les paramètres caractéristiques du substrat, de la bicouche puis de la double bicouche. Cette technique permet alors d'obtenir trois paramètres caractéristiques du système :

1. la densité de longueur de diffusion $\rho$ caractéristique de l'espèce atomique ;

2. l'épaisseur D de la couche ;

3. une largeur d'interface $\sigma$ entre couches d'espèces atomiques différentes généralement prise en compte par un facteur de type Debye-Waller (voir cours G. Fragneto).

La signification physique des deux premiers paramètres est relativement claire (même si leur interprétation n'est pas toujours évidente). Pour le troisième paramètre les choses sont plus complexes. L'interface entre deux espèces chimiquement différentes n'est jamais parfaitement définie à l'échelle moléculaire. D'autre part, différents phénomènes physiques peuvent contribuer à créer un élargissement de la largeur apparente de l'interface. On peut citer les rugosités statiques (substrat solide, polymère vitreux...), les fluctuations dynamiques (fluctuations d'interfaces, protusion des lipides, fluctuations de chaînes de polymère), variation de concentration d'une espèce en solution au voisinage d'une interface... On voit donc que l'interprétation des largeurs d'interface peut être extrêmement ardue. En particulier, il faut noter que ce paramètre correspond à une valeur moyenne intégrée sur toutes les échelles spatiales de l'ensemble de ces contributions ce qui complique encore l'analyse. L'idéal est de pouvoir mesurer le spectre de rugosité de l'interface, c'est-à-dire la largeur de chaque mode de vecteur d'onde $q$.

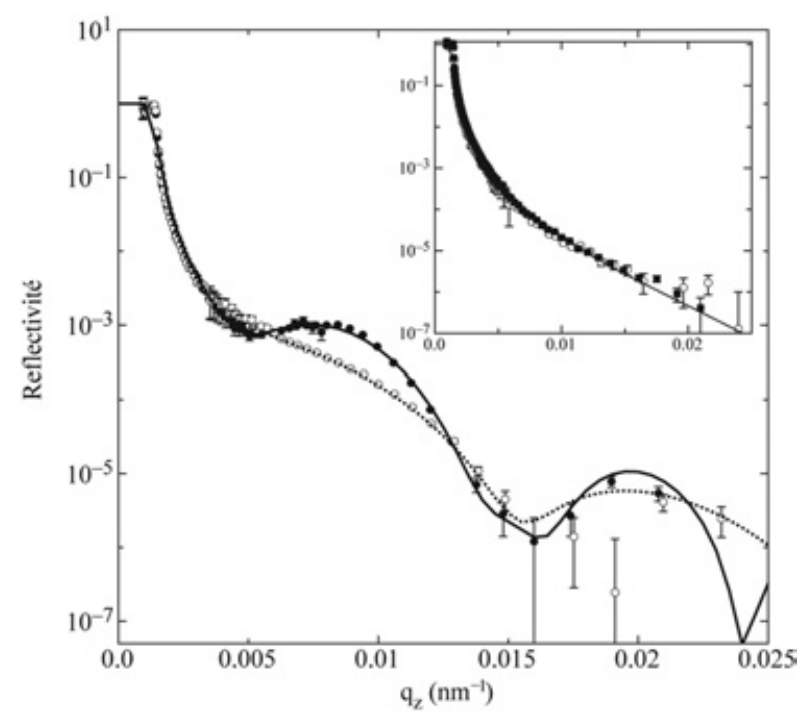

Figure 11. Courbes de réflectivité spéculaire obtenues par diffusion de neutrons (expérience réalisées à I'ILL sur D16 et D17 [52]) ; (०) bicouches de DSPC dans le $\mathrm{D}_{2} \mathrm{O} ;(\bullet)$ double bicouches de DSPC dans le $\mathrm{D}_{2} \mathrm{O}$. En insert on a représenté la courbe de réflectivité obtenue pour un bloc de silicium nu. 
Dans ce qui suit nous allons essayer de montrer quelles informations peuvent être déduites des expériences de diffusion de neutrons et de rayons $\mathrm{X}$ sur les fluctuations d'une membrane supportée. Donnons tout d'abord quelques ordres de grandeurs. Pour une membrane phospholipidique près d'un substrat, le spectre de fluctuations est donné par l'équation qui conduit à l'amplitude moyenne :

$$
\sigma^{2}=\left\langle|u|^{2}\right\rangle=\int d^{2} \vec{q}\left\langle\left|u_{q}\right|^{2}\right\rangle
$$

On peut essayer d'estimer $\sigma$ à partir des ordres de grandeurs suivants :

- $U^{\prime \prime} \simeq 10^{12}-10^{13}$ en phase fluide ;

- $\gamma \simeq 10^{-4}-10^{-5} \mathrm{~N} \cdot \mathrm{m}^{-1}$;

- $\kappa \simeq 10-50 k_{B} T$ en phase fluide ;

On obtient alors une amplitude de fluctuations $\sigma<1 \mathrm{~nm}$, ce qui donne une idée de la difficulté de l'étude!

\section{2 Étude indirecte des fluctuations : gonflement géant}

Les lipides présentent différentes phases bidimensionnelles en fonction de la température. On distingue généralement une phase cristalline à très basse température, une phase gel dans un domaine intermédiaire et une phase fluide à haute température. Dans la phase cristalline, les lipides s'arrangent sur un réseau périodique bidimensionnel et les chaînes possèdent un ordre d'orientation. Dans la phase gel, on perd l'ordre de position à longue portée mais les chaînes conservent un ordre orientationnel. Les lipides sont alors libres de diffuser de façon brownienne avec un coefficient de diffusion de l'ordre de $10^{-9} \mathrm{~cm}^{2} \mathrm{~s}^{-1}$. Finalement, dans la phase fluide, on perd tout ordre de position et d'orientation à longue portée. On a affaire à un fluide bidimensionnel avec des cœefficients de diffusion $\sim 10^{-7} \mathrm{~cm}^{2} \mathrm{~s}^{-1}$.

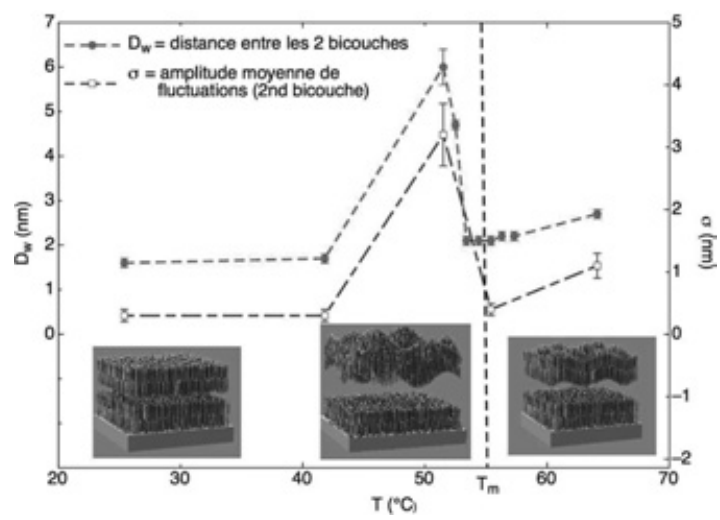

Figure 12. Etude en température d'une double bicouche de DSPC par réflectivité de neutrons[19] : $(\bullet$ rouges $)=$ position moyenne de la bicouche flottante $D_{w} ;$ ( $\circ$ bleus) = amplitude moyenne de flctuations $\sigma$ de la bicouche flottante. Les tirets noirs indiquent la température de transition $T_{m}$ pour un système multilamellaire.

La transition entre la phase gel et la phase fluide est appelée main transition et se produit à la température $T_{m}$. On observe pour certains lipides, entre la phase gel et la phase fluide $\left(T_{p}<T_{m}\right)$ une phase dite ripple phase où la membrane présente des ondulations statiques en forme de dents de scies asymétriques. 
La transition gel-fluide est très coopérative, et la capacité calorifique du système présente un pic très marqué à $T_{m}$. Une analyse thermodynamique fine permet de relier la compressibilité surfacique de la membrane $\Delta \kappa_{T}^{\text {area }}$ à la capacité calorifique [53] :

$$
\kappa_{T}^{\text {area }}=\frac{\gamma_{\text {area } T}^{2} T}{A} c_{p}
$$

où $\gamma_{\text {area }}$ est une constante qui relie les variations d'enthalpie $\Delta H$ et d'aire $\Delta A$ à la transition [53]. La compressibilité $\Delta \kappa_{T}^{\text {area }}$ présente donc aussi un maximum prononcé à $T_{m}$ qui est le signe des grandes fluctuations de densité dans la membrane. Ceci a des conséquences importantes sur ses propriétés mécaniques. En effet, on peut relier le module de courbure de la membrane $\kappa$ à la compressibilité $\Delta \kappa_{T}^{\text {area }}$, $\kappa^{-1}=16 \kappa_{T}^{\text {area }} / D^{2}$. On obtient finalement une expression donnant la variation du module de courbure en fonction de la température :

$$
\frac{1}{\kappa}=f \cdot \frac{1}{\kappa^{\text {fluid }}}+(1-f) \cdot \frac{1}{\kappa^{\text {gel }}}+\frac{16 \gamma_{\text {Surface }}^{2} T}{D^{2} A} \Delta c_{p}
$$

où f est la fraction de lipide dans la phase fluide. Cette relation montre que le module de courbure de la membrane lipidique décroît fortement à la transition.

Cette décroissance a des conséquences importantes sur la membrane supportée. Les fluctuations sont grandes et les interactions de Helfrich deviennent fortes. Le paramètre $\beta=\left(k_{B} T\right)^{2} /(H \kappa)$ augmente et la membrane peut s'écarter du substrat.

La Figure 12 montre l'effet du passage de la phase gel à la phase fluide pour une quadricouche de DSPC. On a reporté la variation de la distance entre les deux bicouches $D_{w}$ et du paramètre de Debye-Waller de la seconde bicouche $\sigma_{f}$ en fonction de la température. On observe une augmentation importante de $D_{w}$ et de $\sigma_{f}$ au voisinage de la main transition. Ce gonflement a été observé pour différents types de lipides [54].

La réflectivité ne permet pas de savoir si l'augmentation de $\sigma_{f}$ doit être attribuée à une élévation importante des fluctuations thermiques ou à un effet statique. On peut néanmoins essayer d'interpréter

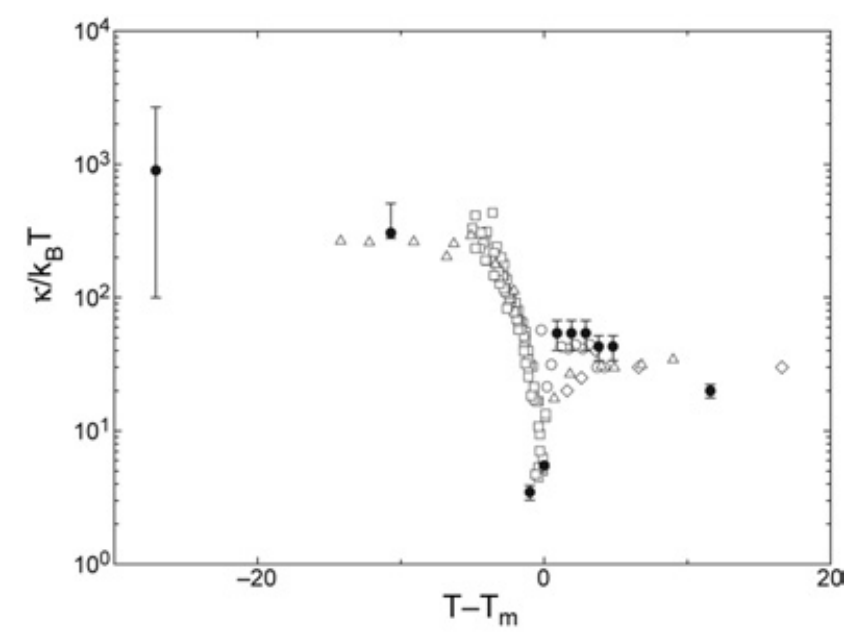

Figure 13. Variation du module de courbure $\kappa$ en fonction de la température pour une bicouche de DSPC : $(\Delta)=$ d'apreès $[17] ;(\circ)=$ d'après $[11] ;(\square)=$ d'apreés $[16] ;(\diamond)=$ d'apreés $[14] ;(\bullet)=$ valeurs déduites des expériences de réflectivité de neutrons sur double bicouche. 
le décollement de la membrane comme un effet de la répulsion de Helfrich. On peut aussi déduire des mesures précédentes une valeur du module de courbure en fonction de la température. On voit sur la Figure 13 que les valeurs obtenues pour $\kappa$ sont en bon accord avec des résultats obtenus avec des techniques macroscopiques sur des vésicules.

Les doubles bicouches sont donc un système modèle intéressant pour l'étude des fluctuations d'une membrane. Mais peut-on aller plus loin et mesurer le spectre de fluctuations de la membrane « quasi-libre » Nous détaillons ce point dans la partie qui suit.

\section{3 Étude directe du spectre de fluctuations par diffusion de rayonnement}

\section{Intensité diffusée}

Dans cette partie nous montrons comment il est possible de mesurer le spectre de fluctuations d'une membrane unique par diffusion de rayonnement. Les expériences décrites ont été réalisées à l'ESRF [55]. On réalise cette étude en couplant à la fois des mesures en géométrie de réflectivité spéculaire et en diffusion hors-spéculaire [20]. Dans ces dernières, le vecteur d'onde de transfert n'a pas seulement une composante $q_{z}$ normale au plan moyen de l'échantillon mais aussi une composante $q_{\|}=\left(q_{x}, q_{y}\right)$ dans le plan de l'échantillon. On s'attend donc à obtenir une information sur la fonction de corrélation de densité de diffuseurs dans le plan $(x, y)$.

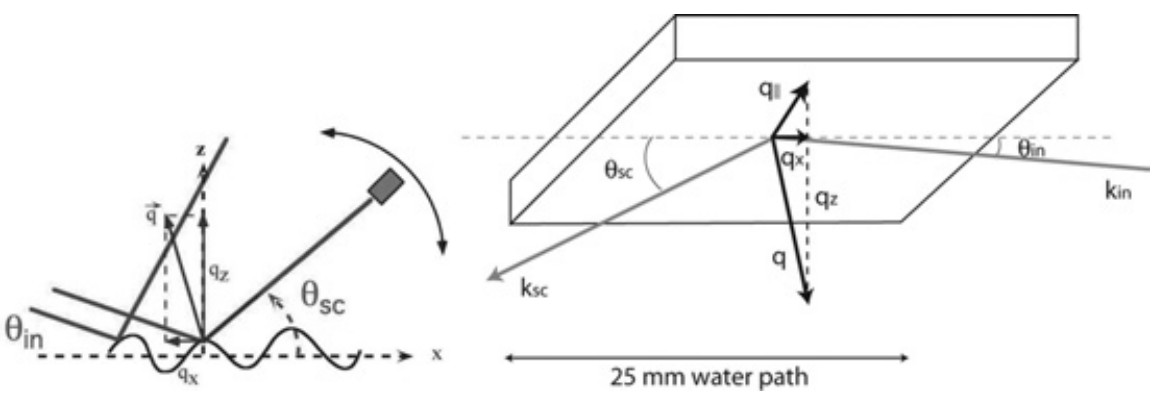

Figure 14. Géométric de la diffusion de rayonment par une surface non parfaitement plane.

On comprend (voir Figure 14) que cette intensité est directement reliée au fait que le système ne présente pas des interfaces planes. D'autres paramètres expérimentaux, comme par exemple la divergence du faisceau incident qui n'est jamais parfaitement parallèle, ou la résolution angulaire du détecteur sont aussi à prendre en considération pour calculer l'intensité diffusée par le système. En particulier, on ne peut plus vraiment parler de direction spéculaire mais plutôt parler de diffusion de rayonnement par la surface. En effet, la direction spéculaire n'est plus vraiment particulière et le calcul de l'intensité diffusée dans cette direction doit être fait avec soin. On en verra un exemple dans ce qui suit. Pour plus de détails, on peut se reporter à l'article de revue de Daillant et Alba [56].

Comme nous allons le voir, le signal diffusé par l'interface est très faible. On a donc intérêt à travailler en incidence rasante. En se plaçant juste en dessous de l'angle de réflexion totale, seule une onde évanescente pénètre dans le substrat et on est sensible uniquement à une couche d'épaisseur comparable à la profondeur de pénétration de l'onde.

La principale difficulté vient alors du faible signal diffusé par l'interface par rapport à la diffusion par le solvant comme on peut le voir sur la Figure 16:

- Les croix noires (+) sont le signal brut mesuré sur une bicouche-OTS de DSPC. On constate que le signal diffusé décroît extrêmement rapidement puisque dès les tous petits angles on détecte environ 1 photon sur un million $\left(I / I 0 \sim 10^{-6}\right)$. 


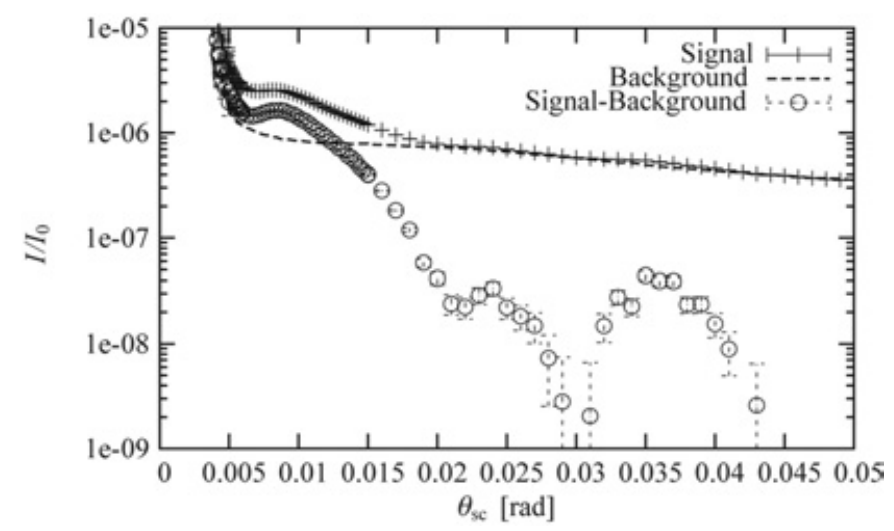

Figure 15. Mesure de reflectivité hors-spéculaire : $(+)=$ signal total diffusé ; $(-)=$ signal diffusé par le volume ; $(\circ)=$ après soustraction du bruit de fond, signal diffusé par l'interface.

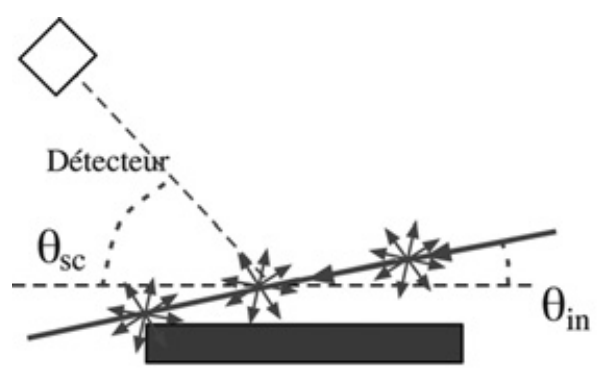

(a)

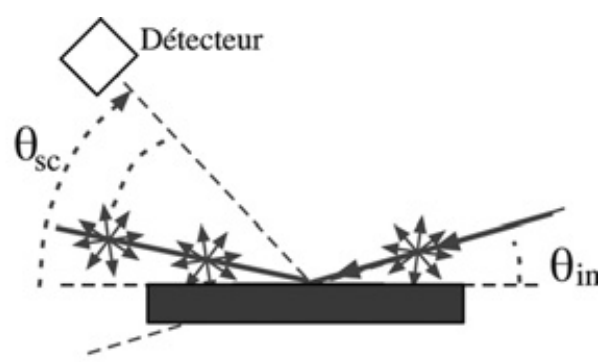

(b)

Figure 16. Principe de la mesure du « bruit de fond » dû à la diffusion par l'eau : l'échantillon est décalé du faisceau (schéma (a)), et la diffusion dans la géométrie expérimentale (schéma (b)) peut-être déterminée grâce à la relation 28.

- La courbe en tirets $(-)$ correspond au signal $I_{m}$ mesuré expérimentalement pour la diffusion par le volume d'eau traversé pour l'angle d'incidence $\theta_{i n}$ en fonction de $\theta_{s c}$. Elle est mesurée après avoir retiré l'interface du faisceau incident. Elle permet d'obtenir le signal de « bruit de fond » $I_{B}$ diffusée par le volume d'eau dans la géométrie de l'expérience à partir de l'équation 28 :

$$
I_{B}\left(\theta_{s c}\right)=\frac{\left[I_{m}\left(\theta_{s c}\right)+R\left(\theta_{i n}\right) \times I_{m}\left(\theta_{s c}-2 \theta_{i n}\right)\right]}{2}
$$

La première partie de $I_{B}$ vient de la diffusion par l'eau du faisceau incident dans la direction $\theta_{s c}$. La seconde contribution $R\left(\theta_{i n}\right) \times I_{m}\left(\theta_{s c}-2 \theta_{\text {in }}\right) / 2$ a pour origine la diffusion par l'eau du faisceau spéculaire réfléchi dans la direction $\theta_{s c}$ (l'angle entre ce faisceau et le détecteur est donc de $\left.\theta_{s c}-2 \theta_{i n}\right)$. Il doit donc être corrigé du coefficient de réflectivité $\mathrm{R}$ que l'on détermine expérimentalement.

- La courbe expérimentale de diffusion par l'interface est obtenue par soustraction des deux courbes précédentes. Elle est donnée par les points (०). 
L'analyse de l'intensité diffusée est compliquée par le fait que l'on travaille en incidence rasante. En effet, les sections efficaces de diffusion sont alors grandes et on ne peut plus négliger les diffusions multiples. L'approximation cinématique classique de Born n'est plus valable et on doit utiliser des méthodes plus raffinées. L'échantillon ne présentant que de faibles variations de densité électronique par rapport à l'eau on peut appliquer une théorie de perturbation comme la méthode DWBA (Distorted Wave Born Approximation) en utilisant comme état de référence le système parfaitement plat [20, 57]. On montre alors que l'intensité diffusée par l'interface est donnée par :

$$
\frac{d \sigma}{d \Omega}=\frac{d \sigma}{d \Omega_{\text {spec }}}+r_{e}^{2}\left|t_{\mathrm{H}_{2} \mathrm{O}, \mathrm{S}_{\mathrm{i}}}\left(\theta_{\text {in }}\right)\right|^{2}\left|t_{\mathrm{H}_{2} \mathrm{O}, \mathrm{S}_{\mathrm{i}}}\left(\theta_{s c}\right)\right|^{2}\left(\vec{e}_{\text {in }} \cdot \vec{e}_{s c}\right)^{2}\left\langle\left|\int d^{3} \vec{r} \delta \rho(\vec{r}) e^{i \vec{q} \cdot \vec{r}}\right|^{2}\right\rangle
$$

où $t_{\mathrm{H}_{2} \mathrm{O}, \mathrm{Si}}\left(\theta_{\text {in }}\right)$ and $t_{\mathrm{H}_{2} \mathrm{O}, \mathrm{Si}}\left(\theta_{s c}\right)$ sont les coefficients de transmission de Fresnel entre l'eau et le substrat pour les angles d'incidence $\theta_{i n}$ et de diffusion $\theta_{s c}$. Le premier coefficient donne avec une bonne approximation le champ électrique diffusé par la densité électronique, le second coefficient indique comment ce champ se propage jusqu' au détecteur. Le terme $\left(\vec{e}_{i n} \cdot \vec{e}_{s c}\right)$ permet de prendre en compte la polarisation du champ électrique.

La perturbation du signal diffusé due à la brisure de l'invariance par translation dans le plan de l'échantillon est donnée par le terme $\left\langle\delta \rho(r) \delta \rho\left(r^{\prime}\right)\right\rangle$. Ces fluctuations de densité électronique peuvent avoir pour origine des variations de composition de la membrane : pores [58], séparation de phase [59]... Elles peuvent aussi être dues à des changements morphologiques de l'interface : rugosité statique [60], protrusions [61], ondulations statiques (phase ripple) [62, 63], fluctuations de forme d'origine thermique [6]...

Dans le cas où on a uniquement des fluctuations de hauteur, on peut aussi exprimer le dernier terme comme [64] :

$$
\begin{aligned}
\frac{d \sigma}{d \Omega} & =\frac{A}{q_{z}^{2}}+r_{e}^{2}\left|t_{\mathrm{H}_{2} \mathrm{O}, \mathrm{S}_{\mathrm{i}}}\left(\theta_{i n}\right)\right|^{2}\left|t_{\mathrm{H}_{2} \mathrm{O}, \mathrm{S}_{\mathrm{i}}}\left(\theta_{s c}\right)\right|^{2}\left(\widehat{e}_{i n} \cdot \widehat{e}_{s c}\right)^{2} \\
& \times\left|\int \rho(z) e^{i q_{z} z} d z\right|^{2} e^{-q_{z}^{2}\left\langle z^{2}\right\rangle} \\
& \times \int d r_{\|}\left(e^{q_{z}^{2}\left\langle z(0) z\left(r_{\|}\right)\right\rangle}-1\right) e^{i q_{\|\cdot r\|}}
\end{aligned}
$$

Dans cette expression on doit faire intervenir les différentes interfaces de notre système. On distinguera donc :

1. l'interface du substrat de silicium $z_{s}(x, y)$;

2. la première couche mixte OTS-lipide $z_{1}(x, y)$;

3. la bicouche fluctuante $z_{2}(x, y)$. on obtient alors :

$$
\begin{aligned}
\frac{d \sigma}{d \Omega}= & \frac{d \sigma}{d \Omega_{\text {ref }}}+r_{e}^{2}\left|t_{H_{2} o, S_{i}}\left(\theta_{i n}\right)\right|^{2}\left|t_{H_{2} o, S_{i}}\left(\theta_{s c}\right)\right|^{2}\left(\widehat{\mathrm{e}}_{i n} \cdot \widehat{\mathrm{e}}_{s c}\right)^{2} \\
& \left\langle\left|\int d \mathrm{r} e^{i \vec{q}_{\|} \cdot \vec{r}_{\|}}\left[\frac{\rho_{S i}-\rho_{\omega}}{i q_{z}} e^{i q_{z} z_{s}\left(\vec{r}_{\|}\right)}+\delta \widetilde{\rho}_{1}\left(q_{z}\right) e^{\left.i q_{z}\left(z_{1}\left(\vec{r}_{\|}\right)\right)\right)}+\delta \widetilde{\rho}_{2}\left(q_{z}\right) e^{\left.i q_{z}\left(z_{2}\left(\vec{r}_{\|}\right)\right)\right)}\right]\right|^{2}\right\rangle,
\end{aligned}
$$

où $\delta \widetilde{\rho}_{1}\left(q_{z}\right)$ et $\delta \widetilde{\rho}_{2}\left(q_{z}\right)$ sont respectivement les profils de densité électronique de la couche mixte lipideOTS (ou de la première bicouche) et de la bicouche libre. Il nous faut donc décrire ces profils de densité électronique, mais aussi se donner des modèles pour calculer les fonctions de corrélation $\left\langle z_{i}(\vec{r}) z_{j}(\vec{r})\right\rangle$ (avec $(i, j)=s, 1,2)$. Le calcul complet de l'intensité diffusée nécessite encore la prise en compte de la résolution expérimentale, c'est-à-dire d'intégrer l'expression précédente sur tous les angles d'incidence et de diffusion accessibles [56]. On ne détaillera pas ici cette opération importante, qui nécessite de bien connaître la géométrie expérimentale. 
Cette approche permet de décrire l'intensité diffusée en fonction de $q_{x}$ et de $q_{y}$. En particulier, on peut l'utiliser pour analyser de façon simultanée les courbes de réflectivité hors-spéculaire $\left(\theta_{\text {in }} \simeq \theta_{c}=C t e\right)$ et les courbes spéculaires $\left(q_{x}=0\right)$. On verra en particulier que cette approche permet de prendre en compte la contribution du diffus à la direction spéculaire liée à la divergence du faisceau incident. cette contribution devient importante à petite échelle [56].

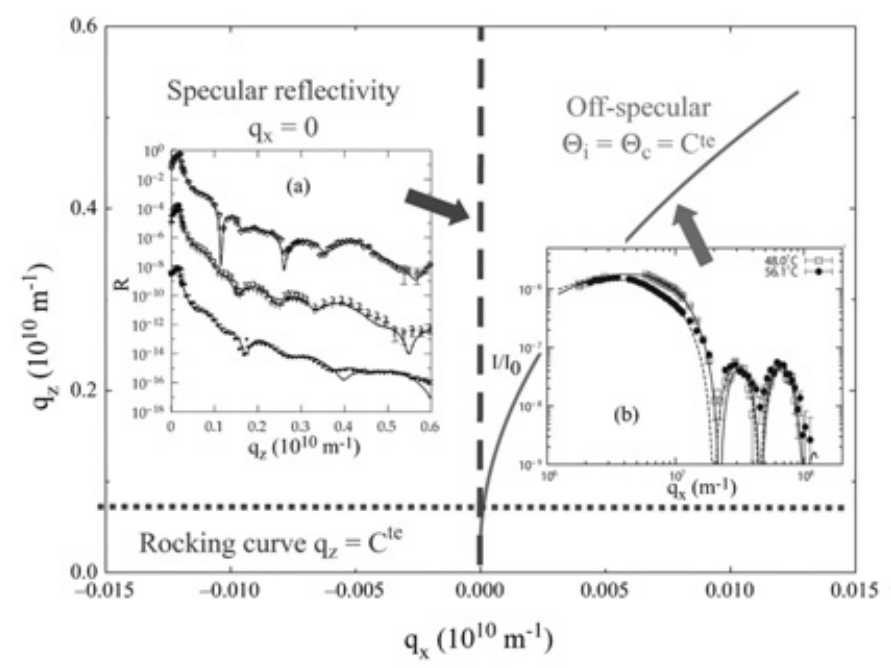

Figure 17. Schéma récapitulant les expériences réalisées dans l'espace réciproque. Les pointillés $(\cdots)$ indiquent les rocking curves qui sont réalisées pour obtenir les points de la courbe spéculaire. La courbe en tirets (- - -) correspond à la réflectivité spéculaire $\left(q_{x}=0\right.$, courbe (a)). La courbe pleine indique le trajet suivi dans les expériences de réflectivité hors-spéculaire $\left(\theta_{i n}=C^{t e}, q_{x}\right.$ et $q_{z}$ varient, courbe (b)).

Description du substrat et de la couche mixte lipide-OTS

Le profil de densité électronique de la couche mixte est décrit par un modèle continu décrit dans la référence [20]. Le substrat de silicium présente une rugosité statique de faible amplitude $(\sigma<5 \AA)$ que l'on peut modéliser en s'inspirant de la description donnée par Palasantzas [60]. On choisit donc comme fonction de corrélation :

$$
\left\langle z_{s}(0) z_{s}\left(r_{\|}\right)\right\rangle=\sigma_{S i}^{2} e^{-\left(\frac{r_{\|}}{\xi_{S i}}\right)^{2 H_{S i}}}
$$

dont on connait une transformée de Fourier approchée :

$$
\left\langle\widetilde{z}_{s}\left(q_{\|}\right) \widetilde{z}_{s}\left(-q_{\|}\right)\right\rangle=2 \pi \frac{\sigma^{2} \xi^{2}}{\left(1+\frac{\left(q_{\|} \xi\right)^{2}}{2 H}\right)^{1+H}}
$$

Sur la Figure 18, sont représentées les données de diffusion hors-spéculaire. Les symboles $(x)$ représentent les résultats obtenus pour les blocs de silicium nu avec le modèle précédent pour $H_{S i}=0.3$, $\sigma_{S i}=0.4 \mathrm{~nm}$ et $\xi_{S i}=6 \mu \mathrm{m}$. L'accord entre les données expérimentales et le modèle est remarquable et cohérent avec les mesures de topographie (rugosité rms et longueur de cohérence) que l'on peut faire par AFM.

On cherche maintenant à décrire la couche mixte OTS-lipide. On peut montrer que la rugosité de cette couche est complètement décorrélée du substrat, ce qui est cohérent avec l'image d'une couche d'OTS assez molle. On suppose alors que le spectre de fluctuations de la couche d'OTS-lipide est donné par une loi comparable à celle du substrat (équations 32 et 33). 


\section{Description de la bicouche fluctuante}

On s'attache maintenant à décrire la bicouche. On va prendre en compte deux contributions différentes pour décrire les écarts de la bicouche par rapport au plan :

1. la bicouche est déposée sur un substrat qui n'est pas parfaitement plan (on vient de décrire son spectre de rugosité) et la membrane doit adapter sa forme à cette rugosité ;

2. la membrane est soumise à l'agitation thermique du solvant et fluctue autour de sa position d'équilibre.

Le premier terme est purement statique. La membrane cherche à adapter sa forme à celle du substrat pour satisfaire le potentiel d'interaction. Pour cela, elle doit se déformer ce qui lui coûte une certaine énergie. La forme statique de la membrane est donc un compromis entre ces deux termes : si le potentiel est très fort, la membrane suit la rugosité du substrat, si le module de courbure ou la tension de surface sont élevés la membrane lisse la rugosité du substrat. Bien sûr la réponse de la membrane dépend fortement de l'échelle spatiale caractéristique de la rugosité. Ce problème a été traité en détail par Swain et al. $[65,66]$ en utilisant la théorie de la réponse linéaire. On montre alors que :

$$
\widetilde{z}_{2, s}\left(q_{\|}\right)=U^{\prime \prime} \frac{\widetilde{z}_{s}\left(q_{\|}\right)}{U^{\prime \prime}+\gamma q_{\|}^{2}+\kappa q_{\|}^{4}}
$$

Les fluctuations thermiques de la membrane autour de cette position d'équilibre sont décrites de façon classique dans l'approximation quadratique (voir équation 7) :

$$
\left\langle z_{t h}\left(q_{\|}\right)^{2}\right\rangle=\frac{k_{B} T}{U^{\prime \prime}+\gamma q_{\|}^{2}+\kappa q_{\|}^{4}}
$$

\section{Discussions des résultats}

A ce stade, tous les outils pour analyser simultanément les données de diffusion spéculaire et horsspéculaire sont en place. Nous allons résumer brièvement les principaux résultats dans cette partie en insistant essentiellement sur la discussion des courbes expérimentales.

Les Figures 18 et 19 donnent un exemple de résultat obtenu pour une bicouche de DSPC en phase gel.

Commençons par discuter la courbe hors-spéculaire. Comme nous l'avons déjà vu, la courbe bleue correspond au spectre de rugosité du substrat. La courbe noire correspond à l'analyse des résultats en prenant uniquement en compte la bicouche fluctuante. On constate qu'elle décrit bien les résultats à grands vecteurs d'ondes où les fluctuations sont dominées par la courbure et la tension de surface. Ce domaine correspond aux résultats présentés dans la référence [20]. A grande échelle on voit que cette description n'est pas suffisante, il faut prendre en compte les corrélations entre la membrane et le substrat via le potentiel d'interaction ce qui correspond à la courbe rouge de la Figure 18.

L'analyse des courbes de réflectivité spéculaire est aussi instructive. Sur la Figure 19, la courbe rouge montre l'analyse du spéculaire de façon classique. L'effet des rugosités statiques ou dynamiques est pris en compte par des facteurs de Debye-Waller. On voit que cette analyse n'est plus suffisante à grands vecteurs d'onde. La courbe verte représente le signal diffusé par les rugosités des interfaces dans la direction spéculaire calculée en prenant en compte la résolution expérimentale. On voit que cette contribution devient importante pour $q_{z}>6 \cdot 10^{9} \mathrm{~m}^{-1}$. Le signal total calculé avec le formalisme précédemment développé et en utilisant les même paramètres que pour la Figure 18 est donné en rouge.

L'analyse des expériences permet donc de caractériser à la fois la structure et le spectre de fluctuations des différentes surfaces : substrat, couche hybride OTS-lipide et bicouche lipidique. L'allure générale du profil de densité électronique caractéristique de la structure du système est donné sur la Figure 20. Une discussion détaillée des paramètres structuraux peut-être trouvée dans la référence [20].

Les valeurs de la tension de surface et du module de courbure ont été mesurées pour différents lipides (DSPC $=$ di- $\mathrm{C}_{18} \mathrm{PC}$ et $\mathrm{DHPC}=$ di- $\mathrm{C}_{17} \mathrm{PC}$ ) et à différentes températures en phase gel et en phase fluide. Les résultats sont résumés sur la Figure 21 et discutés dans la référence [20]. 


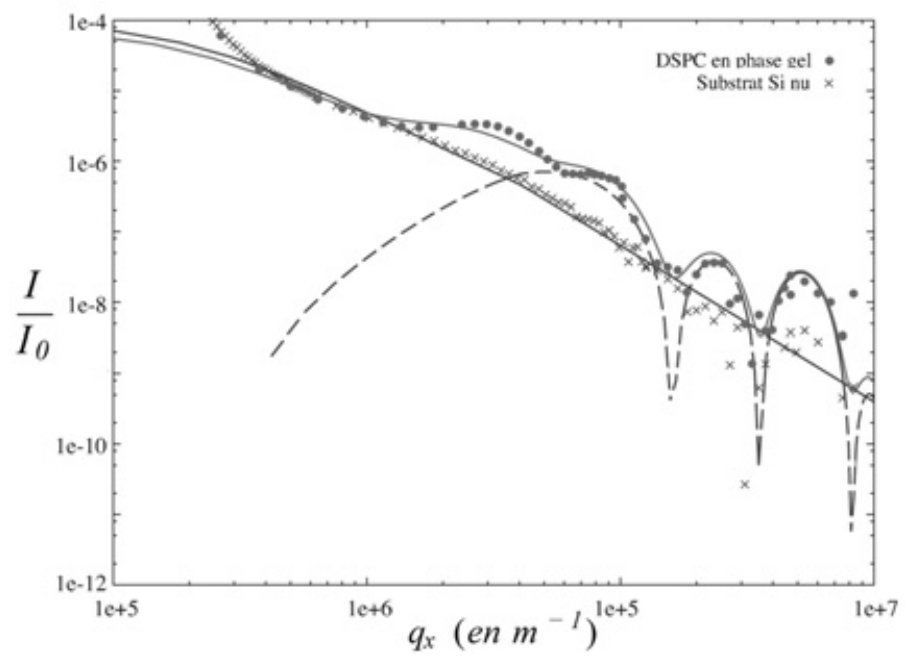

Figure 18. Courbe de réflectivité hors-spéculaire $:(x)=$ Bloc de Silicium $;(\bullet)=$ système mixte OTS-bicouche de DSPC en phase gel $\left(T=49^{\circ} \mathrm{C}\right)$. La courbe bleu correspond à un ajustement des données avec un spectre de rugosité du substrat (équation 32), la courbe noire correspond à un ajustement des données pour une bicouche simple fluctuant avec un spectre du type de l'équation 35 et la courbe rouge prend en compte les corrélations bicouche-substrat.

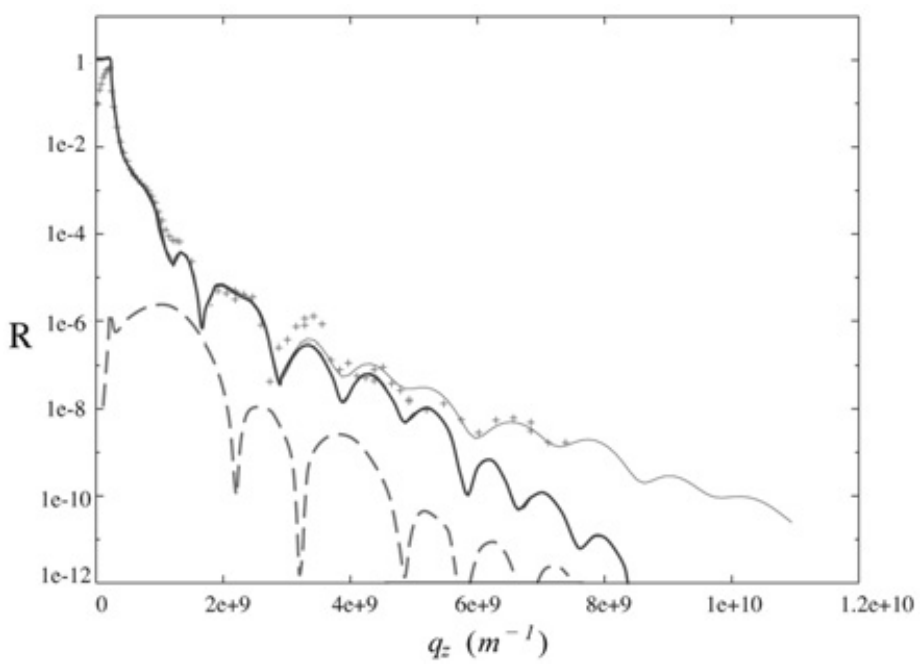

Figure 19. Courbe de réflectivité spéculaire : $(+)=$ système mixte OTS-bicouche de DSPC en phase gel $\left(T=49^{\circ} \mathrm{C}\right)$. La courbe bleu correspond à un ajustement des données en prenant en compte les rugosités avec des facteurs type Debye-Waller, la courbe verte correspond à la contribution dans la direction spéculaire moyenne du diffus due aux fluctuations d'une bicouche simple avec un spectre du type de l'équation 35 et la courbe rouge prend en compte le diffus de manière correcte.

Dans celle-ci, la résolution expérimentale n'était pas suffisante à petit vecteur d'onde $q_{x}$ pour voir correctement les corrélations bicouche-substrat, et ne permettait donc pas d'estimer correctement le potentiel. Comme on l'a vu dans les résultats présentés ici, on peut maintenant accéder à ces corrélations 
et donc mesurer le terme constant dans le spectre de fluctuations [21]. On obtient en phase gel pour du DSPC :

$$
U^{\prime \prime} \sim 10^{12}-10^{13} \mathrm{~J} \cdot \mathrm{m}^{-4}
$$

On peut comparer cette valeur à celles que l'on peut estimer avec un potentiel d'interaction microscopique (équation 14) [36]. On obtient des valeurs de $U^{\prime \prime}$ typiquement comprises entre $3 \cdot 10^{11} \mathrm{~J} . \mathrm{m}^{-4}$ et $2 \cdot 10^{14} \mathrm{~J} . \mathrm{m}^{-4}$, en bon accord avec la valeur expérimentale.

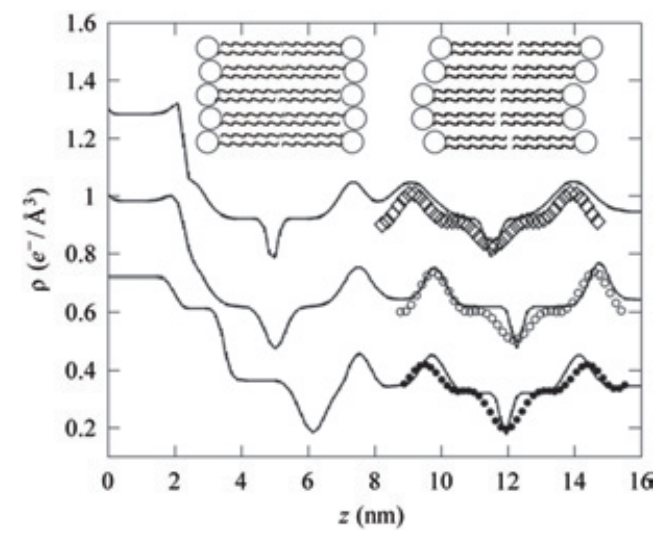

Figure 20. Profil de densité du système obtenu à partir des mesures de diffusion de rayons $X$ : courbe supérieure $(\diamond)$ pour une double bicouche en phase gel, courbe intermédiaire (०) pour double bicouche en phase fluide, courbe inférieure $(\bullet)$ pour une couche mixte OTS-lipide-bicouche en phase fluide (d'après [20]).

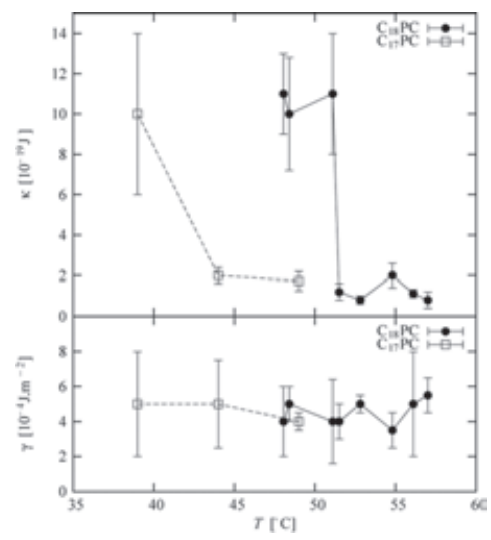

Figure 21. Evolution de la tension de surface $\gamma$ et du module de courbure $\kappa$ en fonction de la température pour une bicouche de DSPC (d'après [20]).

Les techniques de diffusions de rayonnement par une surface fluctuante sont donc très puissantes. Elles permettent de caractériser la structure d'une membrane dans une situation modèle, et de mesurer son spectre de fluctuations dans un domaine submicronique jusqu'alors inaccessible. La Figure 22 schématise les domaines accessibles pour le spectre de fluctuations de la membrane. Néanmoins, elle 
nécessite des flux incidents importants, et ne sont actuellement pas réalisables sur ce type de systèmes avec les neutrons. On peut par contre étudier la diffusion hors-spéculaire de neutron sur des systèmes multilamellaires [67].

\section{CONCLUSIONS}

Ce cours, donné dans le cadre de l'Ecole thématique "Surfaces, interfaces et milieux confinés par diffusion de neutrons", avait pour objectifs d'illustrer à partir de quelques exemples comment la diffusion de rayonnement (neutrons et rayons $X$ ) pouvait apporter des informations sur la physique des membranes de tensio-actifs et plus particulièrement sur leurs fluctuations.

La partie III a été consacrée aux techniques de diffraction de rayonnement par des phases multilamellaires. Elles donnent des informations importantes sur la structure des systèmes lamellaires, mais permettent aussi de mieux comprendre leurs fluctuations. Néanmoins, l'absence d'ordre à longue portée transforme les pics de Braggs en singularités algébriques (lois de puissance) qui rendent difficile l'étude des fluctuations, en particulier dans le cas de systèmes rigides comme les membranes phospholipidiques.

Dans la partie IV, sont décrits deux exemples d'études sur membranes supportées. Dans le premier cas (chapitre IVB), la réflectivité de neutrons est utilisée pour observer le décollement dû au «ramollissement » de la membrane à la transition gel/fluide. Dans la suite (chapitre IVC), sont présentés des résultats plus récents où le spectre de fluctuations d'une membrane unique est mesuré par diffusion de rayons $\mathrm{X}$.

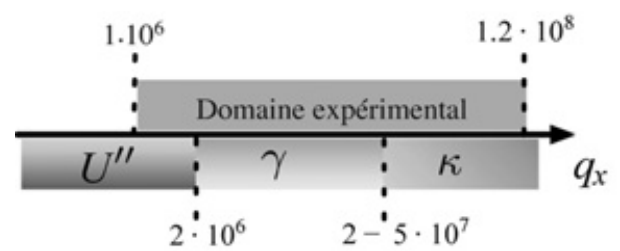

Figure 22. Domaine des valeurs de $q_{x}$ actuellement accessibles dans les expériences de réflectivité hors-spéculaire de rayons $X$ : on a représenté les domaines où les fluctuations sont dominées respectivement par le potentiel, la tension de surface et le module de courbure.

\section{Remerciements}

Les auteurs souhaitent remercier Alan Braslau, Edith Bellet-Amalric, Giovanna Fragneto, Françcois Graner, Klaus Mecke, Serge Mora, François Rieutord et Barry Stidder pour leur participations aux projets sur les membranes supportées. T. C. remercie les organisateurs de l'Ecole thématique « Surfaces, interfaces, milieux confinés par diffusion de neutrons » et plus particulièrement Annie Brulet pour son aide lors de la préparation du manuscrit.

\section{Références}

[1] Katsaras and Gutberlet, Lipid Bilayers, Biological Physics Series (Springer, 2000).

[2] Mouritsen, O., and Andersen, O., eds., In search of a new biomembrane model, Vol. 49 (Copenhagen, 1998).

[3] Nagle, J., and Tristram-Nagle, S., BBA Biomembranes 1469, 159 (2000).

[4] Seifert, U., Advances in Physics 46, 13 (1997).

[5] Sackmann, E., Science 271, 43 (1996).

[6] Helfrich, W., Zeitschrift für Naturforschung 33, 305 (1978).

[7] Safinya, C. R., Roux, D., Smith, G. S., Sinha, S. K., Dimon, P., Clark, N. A. and Bellocq, A. M., Physical Review Letters 57, 2718 (1986). 
[8] Constantin, D., Mennicke, U., Li, C. and Salditt, T., European Physical Journal E 12, 283 (2003).

[9] Pécréaux, J., D. H. G., Prost, J., Joanny, J. F. and Bassereau, P., European Physical Journal E 13, 277 (2004).

[10] Kummrow, M. and Helfrich, W., Physical Review A 44, 8356 (1991).

[11] Mishima, K., Nakamae, S., Ohshima, H. and Kondo, T., Chemistry and Physics of Lipids 110, 27 (2001).

[12] Brochard, F. and Lennon, J.-F., Journal de Physique (Paris) 36, 1035 (1975).

[13] Evans, E. and Rawicz, W., Physical Review Letters 64, 2094 (1990).

[14] Méléard, P., Gerbaud, C., Pott, T., Fernandes-Puente, L., Bivas, I., Mitov, M., Dufourcq, J. and Bothorel, P., Biophysical Journal 72, 2616 (1997).

[15] Dbereiner, H.-G., Evans, E., Kraus, M., Seifert, U. and Wortis, M., Physical Review E 55, 4458 (1997).

[16] Dimova, R., Pouligny, B. and Dietrich, C., Biophysical Journal 79, 340 (2000).

[17] Lee, C.-H., Lin, W.-C. and W. J, Physical Review E 64, 020901 (2001).

[18] Larche, F. C., Appell, J., Porte, G., Bassereau, P. and Marignan, J., Physical Review Letters 56, 1700 (1986).

[19] Fragneto, G., Charitat, T., Graner, F., Mecke, K., Perino-Gallice, L. and Bellet-Amalric, E., Europhysics Letters 53, 100 (2001).

[20] Daillant, J., Bellet-Amalric, E., Braslau, A., Charitat, T., Fragneto, G., Graner, F., Mora, S., Rieutord, F. and Stidder, B., The Proceding of the National Academy of Sciences USA 102, 11639 (2005).

[21] Lecuyer, S., Bellet-Amalric, E., Braslau, A., Charitat, T., Daillant, J., Fragneto, G. and Graner, F., in Preparation (2006).

[22] Ballet, P. and Graner, F. F., European Journal of Physic 27, 951 (2006).

[23] Auvray, L., Charvolin, J. and Di Micoli, J.

[24] Porte, G., cours sur les sytèmes moléculaires organisés, Ecole d'été, Les Houches 1996, non publié.

[25] Seddon, J. and Templer, R., Structure and Dynamics of Membranes - from Cells to Vesicles, Vol. 1A (Springer, 1995).

[26] Canham, P., J. Theor. Bio. 26, 61 (1970).

[27] Helfrich, W., Zeitschrift für Naturforschung 28, 693 (1973).

[28] Willmore, T., Total curvature in Riemannian geometry, 1st ed. (NY-Brisbane-Chichester-Toronto, 1982).

[29] Helfrich, W., Zeitschrift für Naturforschung 28c, 510 (1974).

[30] Peliti, L. and Leibler, S., Physical Review Letters 54, 1690 (1985).

[31] David, F. and Leibler, S., Journal de Physique II France 1, 959 (1991).

[32] Sornette, D. and Ostrowsky, N., Micelles, Membranes, Microemulsions, and monolayers (Springer, 1994).

[33] Michalet, X., Ph.D. thesis, Université Paris VII (1994).

[34] Morse, D., Physical Review E 50, 2423 (1994).

[35] Seifert, U., Physical Review Letters 74, 5060 (1995).

[36] Lipowsky, R., Handbook of Biological Physics, Vol. 1 (Elsevier, 1995).

[37] Lipowsky, R. and Leibler, S., Physical Review Letters 56, 2541 (1986).

[38] Mecke, K. R., Charitat, T. and Graner, F., Langmuir 19, 2080 (2003).

[39] Roux, D. and Bellocq, A. M., Physics of Amphiphiles (North-Holland , Amsterdam, 1985). 
[40] Nallet, J. F., Roux, D. and Prost, J., Physical Review Letters 62, 276 (1989), URL http://link.aps.org/ abstract/PRL/ v62/p276.

[41] Taulier, N., Ph.D. thesis, Université Paris VI (1999).

[42] Landau, L. and Lifschitz, E., Theory of elasticity (Pergamon Press, New York, 1959).

[43] Caillé, A., Comptes Rendus de l'Académie des Sciences Paris pp. 891-893 (1972).

[44] de Gennes, P. G., and Prost, J., The Physics of Liquid Crystals (Oxford University Press, 1993).

[45] Salditt, T., Journal of Physics : Condensed Matter 17, 287 (2005).

[46] Vogel, M., Munster, C., Fenzl, W. and Salditt, T., Physical Review Letters 84, 390 (2000).

[47] Salditt, T., Münster, C., Mennicke, C., Ollinger, U. and Fragneto, G., Langmuir 19, 7703 (2003).

[48] Rädler, J., Feder, T. J., Strey, H. H. and Sackmann, E., Physical Review E 51, 4526-4536 (1995).

[49] Naumann, C. A., Prucker, O., Lehmann, T., Rühe, J., Knoll, W. and Frank, C. W., Biomacromolecules 3, 27 (2002).

[50] Hughes, A. V., Goldar, A., Gestenberg, M. C., Roser, S. J. and Bradshaw, J., Physical Chemistry Chemical Physics 4, 2371 (2002).

[51] Charitat, T., Bellet-Amalric, E., Fragneto, G. and Graner, F. European Physical Journal B 8, 583 (1999).

[52] D17, reflectometer at the ILL, http://www.ill.fr/YellowBook/D17/.

[53] Heimburg, T., Bioch. Biophys. Acta 1415, 147 (1998). '

[54] Fragneto, G., Charitat, T., Bellet-Amalric, E., Cubitt, R. and Graner, F., Langmuir 19, 7695 (2003).

[55] European Synchotron Research Facilities, Beam line BM 32, http://www.esrf.fr/exp_facilities/ BM32/index.htm.

[56] Daillant, J. and Alba, M., Reports on Progress in Physics 63, 1725 (2000).

[57] Daillant, J. and Sentenac, A., in X-ray and Neutron Reflectivity : Principles and Applications, edited by J. Daillant and A. Gibaud (Springer-Verlag, Heidelberg, 1999), Vol. m58 of Lecture Notes in Physics, pp. 121-162.

[58] Bassereau, P. and Pincet, F., Langmuir 13, 7003 (1997).

[59] Heimburg, T., Planar Lipid Bilayers (BLMs) and their Applications (Elsevier, Amsterdam., 2003), chap. Coupling of chain melting and bilayer structure : domains, rafts, elasticity and fusion, pp. 269-293.

[60] Palasantzas, G., Physical Review B 48, 14472 (1993), URL http://prola.aps.org/thumbnail/PRB/v48/ i19/p14472_1? start=0.

[61] Israelachvili, J. N. and Wennerstrom, H., Journal of Physical Chemistry 96, 520 (1992).

[62] Rand, R. P., Chapman, D. and Larsson, K., Biophysical Journal 15, 1117-1124 (1975).

[63] Heimburg, T., Biophysical Journal 78, 1154 (2000).

[64] Mora, S., Ph.D. thesis, Université Paris 11 (2003).

[65] Swain, P. S. and Andelman, D., Langmuir 15, 8902 (1999).

[66] Swain, P. S. and Andelman, D., Physical Review E 63, 51911 (2001).

[67] Münster, C., Salditt, T., Vogel, M., Siebrecht, R. and Peisl, J., Europhysics Letters 46, 486 (1999). 